\title{
Autoimmunity in Wiskott-Aldrich Syndrome: Updated Perspectives
}

\author{
Murugan Sudhakar \\ Rashmi Rikhi \\ Sathish Kumar Loganathan \\ Deepti Suri \\ Surjit Singh (iD)
}

Department of Pediatrics, Advanced Pediatrics Center, Postgraduate Institute of Medical Education and Research, Chandigarh, India
Correspondence: Deepti Suri

Department of Pediatrics, Advanced

Pediatrics Centre, Postgraduate Institute of Medical Education and Research,

Chandigarh, 160012, India

Email surideepti@gmail.com

\begin{abstract}
Wiskott-Aldrich syndrome (WAS) is an uncommon X-linked combined-immunodeficiency disorder characterized by a triad of thrombocytopenia, eczema, and immunodeficiency. Patients with WAS are also predisposed to autoimmunity and malignancy. Autoimmune manifestations have been reported in $26 \%-72 \%$ of patients with WAS. Autoimmunity is an independent predictor of poor prognosis and predisposes to malignancy. Development of autoimmunity is also an early pointer of the need for hematopoietic stemcell transplantation. In this manuscript, we have collated the published data and present a narrative review on autoimmune manifestations in WAS. A summary of currently proposed immunopathogenic mechanisms and genetic variants associated with development of autoimmunity in WAS is also included.
\end{abstract}

Keywords: thrombocytopenia, vasculitis, genetics, hematopoietic stem-cell transplant, bleeding, malignancy

\section{Introduction}

Wiskott-Aldrich syndrome (WAS) is an uncommon X-linked combined immunodeficiency disorder that has a heterogeneous clinical spectrum. ${ }^{1-3}$ Manifestations vary from a relatively milder form of the disease (intermittent X-linked thrombocytopenia [XLT]) characterized by thrombocytopenia with little or no immunodeficiency to severe WAS, with profound immunodeficiency, bleeding episodes, autoimmunity, and increased risk of malignancy. ${ }^{3-5}$ Many patients with WAS have intermediate grades of severity. It is this heterogeneity in the clinical spectrum that makes the initial diagnosis of WAS so challenging.

In a US study, WAS incidence was reported to be 3.3-5.2 per million live male births. ${ }^{6}$ National registry data on primary immunodeficiency diseases from Sweden and Switzerland estimated WAS incidence to be 3.7 and 4.1 per million live births, respectively. ${ }^{7,8}$

In 1994, almost six decades after the initial description of the condition, Derry et al identified the gene responsible for the defect: ${ }^{9}$ WAS, which comprises 12 exons and is located on the short arm of the X chromosome (Xp11.23). ${ }^{10}$ It encodes for WAS protein (WASp), a 502-amino acid cytosolic protein and a key molecule for actin-cytoskeleton polymerization. ${ }^{11-14}$ WASp is ubiquitously expressed in all nonerythroid hematopoietic cells. ${ }^{15}$ It consists of a pleckstrin homology (PH) domain and an enabledvasodilator-stimulated phosphoprotein homology (EVH1, also known as WH1) domain at the amino terminal, a short basic domain (B), a Cdc42- and Rac-interactive binding (CRIB) domain, a large proline-rich region 


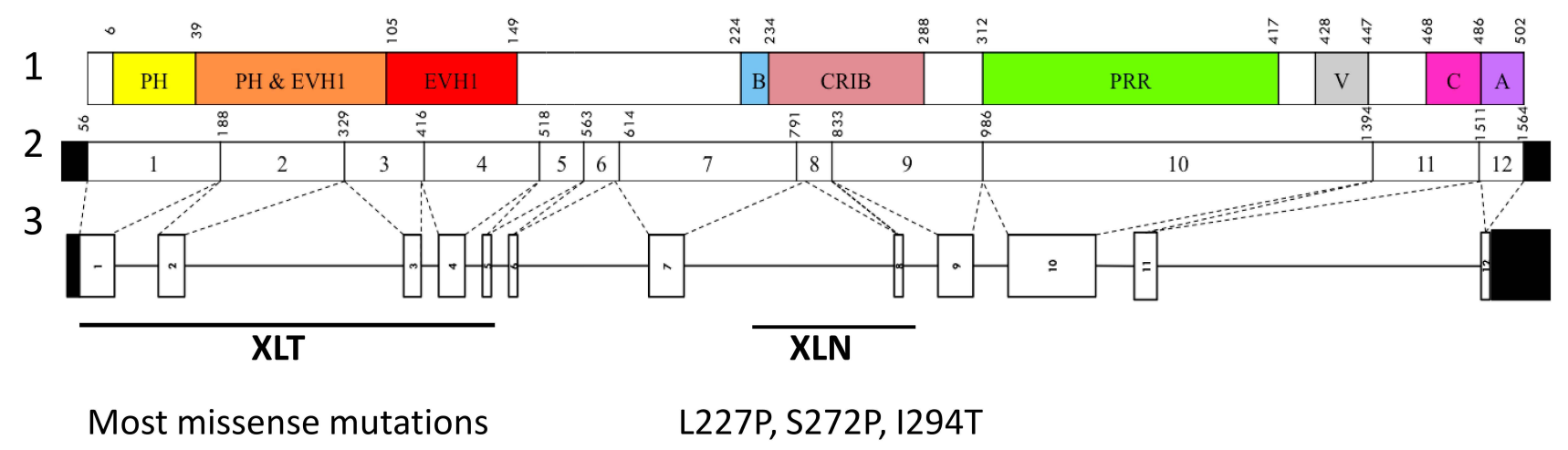

\section{WAS}

Nonsense mutations, insertions, deletions, complex mutations

Figure I Structure of WAS gene, cDNA transcript, and Wiskott-Aldrich syndrome protein. Lane I, Wiskott-Aldrich syndrome protein structure; lane 2, WAS cDNA structure and positions; lane 3, WAS gene structure. Variants associated with XLN: L227P, S272P, and I294T.

Abbreviations: PH, pleckstrin homology; EVHI, vasodilator-stimulated phosphoprotein homology; B, basic; CRIB, Cdc42- and Rac-interactive binding; PRR, proline-rich region; V, verprolin; C, central; A, acidic; XLT, X-linked thrombocytopenia; XLN, X-linked neutropenia; WAS, Wiskott-Aldrich syndrome.

(PRR), and a verprolin/central/acidic (VCA) domain at the carboxyl terminal. (Figure 1)

WAS mutations affect actin cytoskeleton-dependent cellular processes, immunological synapse formation, ${ }^{16-}$ ${ }^{20}$ cell migration, and signaling, ${ }^{21,22}$ and result in impaired functioning of WASp, causing XLT/WAS. Gain-of-function mutations $W A S$ gene manifest as severe congenital Xlinked neutropenia, which is characterized by recurrent bacterial infections, neutropenia, and monocytopenia without thrombocytopenia. ${ }^{23,24}$

The clinical course of WAS is complicated by frequent bleeding episodes, eczema, and recurrent infections. Patients with WAS are also predisposed to autoimmune manifestations and malignancies. ${ }^{3,4}$ Autoimmunity is an independent independent predictor of poor prognosis and predisposes to malignancy. ${ }^{25}$ As such, development of autoimmunity is an early indicator of the need for hematopoietic stem-cell transplantation (HSCT) in patients with XLT/WAS. Though HSCT is curative, it does not completely ameliorate the risk of later development of autoimmunity in WAS. Recent reports on the emergence of postHSCT autoimmunity in these patients have rekindled interest in this field, and suggest the need for a better understanding of autoimmunity. ${ }^{26-30}$ Despite being one of the earliest immunodeficiency syndromes described in the literature, pathophysiological mechanisms of underlying autoimmunity in WAS are still unclear. $^{31}$ This review focuses on putative pathogenetic mechanisms, genetic predisposition, and clinical manifestations of XLT/WAS with autoimmunity.

\section{Methods}

We carried out a literature search on PubMed, Scopus, Web of Knowledge, Google, and Google Scholar using the keywords "Wiskott Aldrich syndrome," "autoimmunity," "eczema," "anemia," "nephritis," "arthritis," "neutropenia," "vasculitis," "Wiskott Aldrich syndrome protein," and "malignancy." A supplementary manual search to identify additional primary studies was conducted and papers published up to December 2020 collated. Data on autoimmune manifestations were extracted from all single/multicenter cohorts and clinical case reports.

Demographic details, clinical and genetic profiles, WAS clinical scores, WASp expression, and outcomes were tabulated.

\section{Incidence of Autoimmune Manifestations}

One of the earliest reports of autoimmunity in WAS was published in 1976 by Gershwin et al, who evaluated patients with primary immunodeficiency diseases for the presence of autoantibodies. $^{32}$ Three of eleven children with WAS had autoantibodies against nucleic acids. However, a US multicentre study of a cohort of patients with WAS made no mention of autoimmunity. ${ }^{6}$ 
In 1994, Sullivan et al reported autoimmune manifestations in $40 \%$ of patients with WAS. ${ }^{25}$ The study highlighted autoimmunity to be a risk factor of the development of malignancy. Since then, autoimmunity has been a well-recognized entity in patients with WAS and an important indicator in predicting clinical outcomes and prognoses. Subsequently, several studies from different centers have reported variable figures $(26 \%-72 \%)$ for the occurrence of autoimmune manifestations in patients with WAS (Table 1). ${ }^{25,28-30,33-40}$

\section{Proposed Mechanisms of Autoimmunity}

WASp is involved in cytoskeleton remodeling, and absence of or residual WASp-expression defects cause- functional defects in all immune-system cells. Formation of immunological synapses in T cells and T-cell receptor (TCR)-dependent activation is impaired in WAS. ${ }^{41-43}$ Reduced cytotoxic activity of $\mathrm{T}$ cells, natural killer cells, and naturally occurring regulatory $\mathrm{T}\left(\mathrm{nT}_{\text {reg }}\right)$ cells contributes to poor pathogen clearance. ${ }^{41-45}$ Motility, adhesion, and migration of $\mathrm{B}$ cells is also defective. ${ }^{45}$ However, underlying mechanisms for occurrence of autoimmune manifestations are still not completely understood. Some hypotheses currently proposed for development of autoimmunity in WAS are summarized in the following sections.

\section{Role of T Cells in Autoimmunity in WAS}

Autoimmunity is caused by failure in self-tolerance mechanisms. $T_{\text {reg }}$ cells play a pivotal role in immunotolerance and prevent autoimmunity. They prevent autoimmunity by maintaining tolerance to self-antigens and suppression of excessive immunoresponse. Development and function of $\mathrm{T}_{\text {reg }}$ cells requires effective TCR signaling with involvement of the CD28 costimulator, expression of master regulator FOXP3, and growth maintenance by IL2. ${ }^{46}$ Although peripheral blood $\mathrm{T}_{\text {reg-cell numbers have been found to be comparable in }}$ patients with WAS and healthy controls, WASp-deficient $\mathrm{T}_{\text {reg }}$ cells demonstrated impaired ability to suppress proliferation of activated T-effector cells. ${ }^{47,48}$ Distribution and phenotype of $\mathrm{nT}_{\text {reg }}$ cells have also been found to be normal in the thymi and spleens of WASp-deficient mice. ${ }^{49} \mathrm{nT}_{\text {reg }}$ cells, however, have been found to be reduced in inflamed peripheral tissue and lymph nodes. Reduction in $\mathrm{nT}_{\text {reg }}$ cells correlates with lack of tissue-homing markers like integrin $\mathrm{a}_{4} \beta_{7}$, chemokine receptor CCR4, and P- and E-selectin ligands. ${ }^{44}$

A mouse model of autoimmunity has failed to control aberrant T-cell activation by WASp-deficient $\mathrm{nT}_{\text {reg }}$ cells. ${ }^{45}$
WASp-deficient $\mathrm{nT}_{\text {reg }}$ cells fail to suppress B-cell activation and proliferation. Defective granzyme-mediated B-cell killing by $\mathrm{nT}_{\text {reg }}$ cells has been demonstrated in some studies. ${ }^{45,47}$

\section{Role of B Cells in Autoimmunity in WAS}

The role of $B$ cells and autoantibodies in the pathogenesis of autoimmune diseases like lupus is well recognized. Bcell dysfunction in patients with WAS is evident from the variable distribution of serum immunoglobulins and demonstration of autoantibodies. Classically, WAS is associated with low serum IgM, normal IgG, and elevated IgE and IgA levels. Patients have impaired response to polysaccharides and other $\mathrm{T}$ cell-independent antigens. ${ }^{3,50}$

Elevated IgM levels correlate with development of autoimmunity. Around $90 \%$ of patients with elevated IgM levels have been found to have developed AIHA in comparison to none with low IgM. ${ }^{33}$

WASp deficiency affects adhesion, motility, and homing of B cells. ${ }^{45,51}$ Defective B-cell function results in insufficient pathogen clearance, chronic immunoactivation, and failure of peripheral B-cell tolerance. In addition, reduced surface expression of complement receptors CD21 (CR2) and CD35 (CR1) results in impaired opsonization and negative selection of self-reactive B cells, thereby breaking peripheral tolerance and helping in autoantibody production. ${ }^{52}$

Murine WASp-deficient B cells demonstrate increased proliferation with autoantibody production and differentiation into plasmablasts. ${ }^{53}$ Enhanced proliferation of transitional $\mathrm{B}$ cells in response to stimulation by antigen or MYD88 has been seen in both humans and mice. ${ }^{54-56}$

$B_{\text {reg }}$ cells influence the balance and recruitment of $T_{\text {reg }}$ cells and $T_{H} 17$ cells during inflammation. Recent studies have suggested that WASp is required for normal $\mathrm{B}_{\text {reg-cell numbers }}$ and functions. ${ }^{57}$ Murine studies have also revealed reduced levels of IL10 secreting $B_{\text {reg }}$ cells (B10) in patients with WAS. $^{44}$

Bouma et al found reduced numbers of IL10-producing $B_{\text {reg }}$ cells, reduced $T_{\text {reg }}$ cells, and increased $T_{H} 17$ cells in arthritic WAS-knockout mice. Adoptive transfer of wildtype $B_{\text {reg }}$ cells ameliorated arthritis and restored the balance between $\mathrm{T}_{\text {reg }}$ and $\mathrm{T}_{\mathrm{H}} 17$ cells. ${ }^{57}$

\section{Role of Invariant NKT Cells in Autoimmunity in WAS}

Invariant NKT cells possess properties of both $\mathrm{T}$ and NK cells. They prevent autoimmunity by limiting development of $\mathrm{T}_{\mathrm{H}} 17$ cells, anti-DNA antibody production, and 


\begin{tabular}{|c|c|c|c|c|c|c|c|c|c|c|c|c|}
\hline 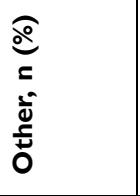 & $\begin{array}{l}\widehat{\sigma} \\
\stackrel{0}{0} \\
\underline{0}\end{array}$ & 1 & 1 & 1 & 1 & 1 & 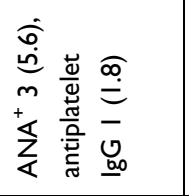 & 1 & 1 & 1 & 1 & 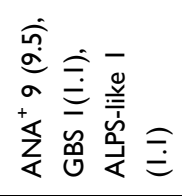 \\
\hline 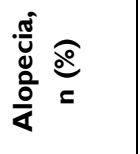 & $\widehat{\cong}$ & 1 & 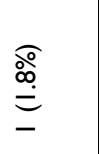 & 1 & $\begin{array}{c}\substack{\infty \\
\stackrel{d}{d}} \\
-\end{array}$ & 1 & 1 & 1 & 1 & $\widehat{\Xi}$ & 1 & 1 \\
\hline$\stackrel{\circ}{\mathrm{O}}$ & 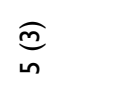 & $\underset{\sim}{\sigma}$ & $\underset{\sim}{\mathbb{E}}$ & 1 & $-\stackrel{\widehat{\infty}}{\dot{n}}$ & 1 & 1 & 1 & 1 & $\underset{\sim}{\widehat{d}}$ & 1 & 1 \\
\hline 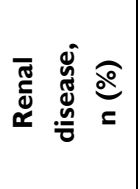 & 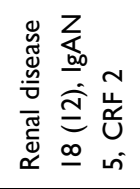 & 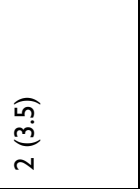 & 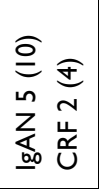 & 1 & 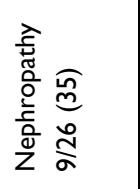 & 1 & 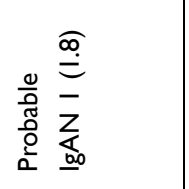 & 1 & । & 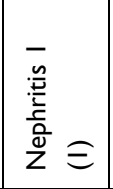 & 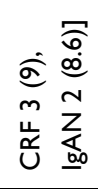 & 1 \\
\hline 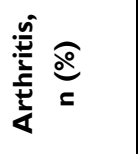 & 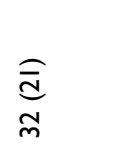 & $\begin{array}{l}\underset{\mathfrak{d}}{0} \\
\underline{0}\end{array}$ & $\underset{m}{\widehat{\theta}}$ & I & 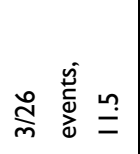 & 1 & $\stackrel{\widehat{o}}{\Xi}$ & 1 & 1 & $\widehat{\Xi}$ & 1 & 1 \\
\hline 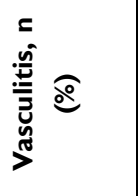 & $\begin{array}{l}\text { F. } \\
\stackrel{0}{0} \\
\stackrel{m}{0}\end{array}$ & $\begin{array}{l}\underset{\text { dิ }}{0} \\
\underline{0}\end{array}$ & $\underset{\sigma}{\widehat{\infty}}$ & I & 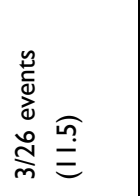 & 1 & 1 & $\underbrace{\widehat{\alpha}}_{\sim}$ & । & $\underset{N}{a}$ & 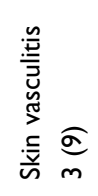 & 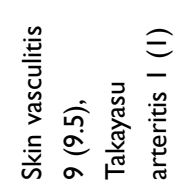 \\
\hline 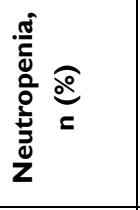 & 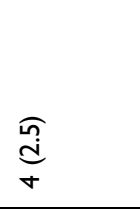 & $\begin{array}{l}\stackrel{\text { I }}{d} \\
\text { 士 }\end{array}$ & 1 & 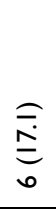 & 1 & 1 & 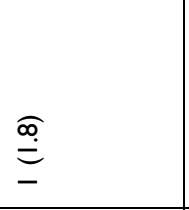 & 1 & 1 & $\frac{n}{n}$ & 1 & 1 \\
\hline 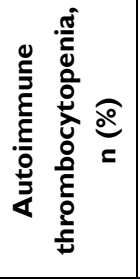 & 1 & $\begin{array}{l}\hat{i} \\
\text { re } \\
\underline{\infty}\end{array}$ & 1 & 1 & 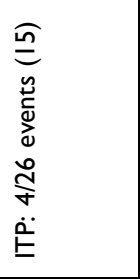 & 1 & 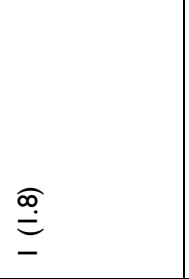 & 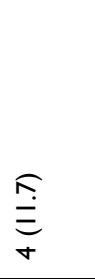 & । & $\begin{array}{l}\text { 年 } \\
\text { a }\end{array}$ & 1 & 1 \\
\hline $\begin{array}{l}\stackrel{0}{8} \\
\leq \\
4 \\
\frac{1}{4}\end{array}$ & 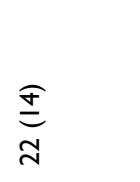 & $\begin{array}{l}\stackrel{\circ}{\circ} \\
\stackrel{N}{\circ}\end{array}$ & $\underset{m}{\widehat{e}}$ & $\underset{0}{\stackrel{\bar{ञ}}{\leftrightarrows}}$ & 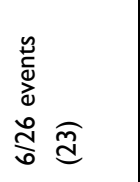 & 1 & 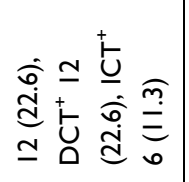 & $\begin{array}{l}\text { f. } \\
\text { di } \\
a\end{array}$ & । & 唫 & 1 & 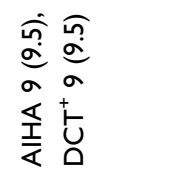 \\
\hline 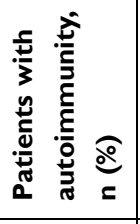 & $\frac{\bar{q}}{\bar{q}}$ & $\begin{array}{l}\widehat{E} \\
\text { S } \\
\end{array}$ & $\begin{array}{l}\underset{d}{d} \\
\text { I }\end{array}$ & 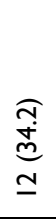 & 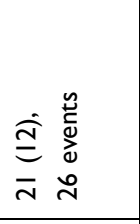 & $\begin{array}{l}\text { ్ㅗ } \\
\text { 으 }\end{array}$ & $\begin{array}{l}\vec{f} \\
\stackrel{d}{d} \\
\underline{ \pm} \\
\end{array}$ & $\underset{\underline{\underline{n}}}{\stackrel{F}{\underline{y}}}$ & $\begin{array}{l}\text { } \\
\stackrel{n}{n} \\
\underline{n}\end{array}$ & 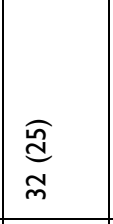 & $\underset{m}{\sigma}$ & $\begin{array}{l}\text { } \\
\infty \\
\infty \\
m\end{array}$ \\
\hline 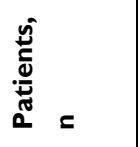 & 吉 & นn & 요 & $\stackrel{\sim}{m}$ & $\begin{array}{l}\underset{\underline{x}}{x} \\
\underline{\underline{x}}\end{array}$ & $\hat{f}$ & กิ & 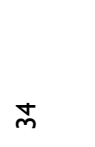 & $\mathcal{F}$ & さે & $\stackrel{\sim}{\sim}$ & $\stackrel{2}{\alpha}$ \\
\hline 窟 & 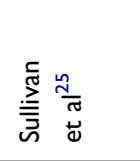 & 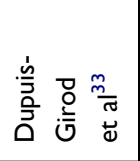 & 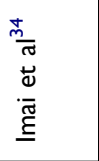 & 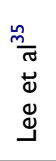 & 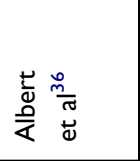 & 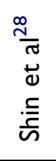 & 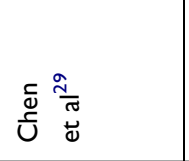 & 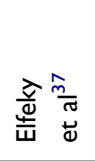 & $\begin{array}{l}\frac{\infty}{0} \\
\stackrel{\frac{0}{0}}{0} \\
\stackrel{\equiv}{\equiv}\end{array}$ & 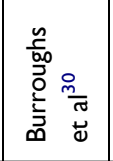 & 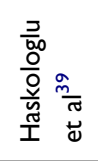 & 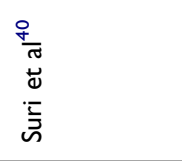 \\
\hline
\end{tabular}


autoreactive B-cell production. ${ }^{58,59}$ Reduced numbers, defective invariant NKT cells, and increased IFN $\gamma$ production have been reported in WASp-deficient mice. ${ }^{60,61}$

\section{Role of IFNI in Autoimmunity in WAS}

Plasmacytoid dendritic cells (pDCs) are specific subsets that produce IFN1 in response to foreign nucleic acids. Viral infections and certain self-nucleic acids serve as stimulants of TLR7 and TLR9 and keep them persistently activated. Susceptibility to viral infections, impaired clearance, and exposure of self-antigens following cell death activate the IFN1 pathway. ${ }^{62}$ This pathogenic mechanism is associated with several autoimmune diseases, such as lupus, Sjögren's syndrome, and psoriasis. ${ }^{62}$

WASp deficiency leads to exaggerated activation of TLR9 by its ligand and subsequent increased IFN1 signature. ${ }^{63}$ WASp-deficient mice show persistent activation of pDCs and elevated IFN1 levels. Ablation of IFN1 in WASp-deficient mice results in blockade of chronic activation of pDCs. This is accompanied by remission of colitis and reduction in spleen size. ${ }^{63}$

\section{Defects in Apoptosis Pathway and Development of Autoimmunity in WAS}

Restimulation-induced cell death is a process that augments Fas-mediated apoptosis of activated $\mathrm{T}$ cells. It helps eliminate $\mathrm{T}$ cells that are activated against chronically expressed antigens like autoantigens. Nikolov et al demonstrated defective FasL in WASp-deficient mice leading to defective elimination of activated $\mathrm{T}$ cells and predisposition to autoimmunity. ${ }^{64}$ Defective Fas has also been hypothesized to be a pathophysiological mechanism for lymphoproliferative lupus-like syndrome in Fas/FasLnegative mice. ${ }^{65,66}$

\section{Role of Inflammasomes in Autoimmunity in WAS}

Excessive inflammasome activation has been shown in human monocytes and mouse DCs. ${ }^{67}$ Some autoimmune manifestations (especially rash and arthralgias) are related to excessive NLRP3-inflammasome activation.

\section{Correlation of Genetic Variants with Autoimmunity}

We compiled details of genetic variants reported in the literature in patients with WAS and autoimmune manifestations. In sum, 166 variants were associated with autoimmunity in 197 WAS patients (Table 2, Figure 2). These included 143 exonic (136 well-defined) and 23 intronic variants. Exonic variants were located at 83 amino acid positions. Most variants were found in the $\mathrm{PH}$ and EVH1 domains $(\mathrm{n}=84,50.6 \%)$ followed by the PRR domain $(n=20,12.2 \%)$, VCA domain $(n=9,5.5 \%)$, and $\mathrm{B}$ and CRIB domains $(\mathrm{n}=4,2.4 \%)$. The variants described included missense (56), deletions (50, 41 exonic and nine intronic), nonsense (27), splicesite (25), insertions (7), unknown frameshift (five), and complex (four).

Deletions are spread across the gene, while most missense variants are seen in the $\mathrm{PH}$ and EVH1 domains. Missense variants (p.T45M and p.T45K) at position 45 in PH and EVH1 have been found to be associated with autoimmunity. Normal/absent WASp expression was found such patients. ${ }^{34,36,75,76}$ Twelve patients with missense variants at position 86 (R86G, R86C, R86H and R86A) developed autoimmunity. Both p.E31K and p. E133K were reported in five patients each that developed autoimmunity.

Patients with XLT can progress to autoimmune WAS. The XLT phenotype with missense variants in PH and EVH1 is most associated with development of autoimmune WAS. Albert et al identified 13 positions associated with XLT to autoimmune WAS progressions, 9 of which lie in $\mathrm{PH}$ and EVH1 domain.

Patients with XLT can also develop autoimmunity. Albert et al identified 13 amino acid positions that were prone to progress to autoimmune WAS. ${ }^{36}$

We identified 18 variants in 25 patients reported to demonstrate progression of XLT to autoimmune WAS. Of these, 13 (all missense) were located in PH and EVH1.

\section{Clinical Manifestations}

\section{Autoimmune Hemolytic Anemia (AlHA)}

AIHA is the commonest autoimmune manifestation seen in patients with WAS, accounting for $30 \%-85 \%$ of all autoimmune manifestations. The reported frequency of AIHA is $8 \%-36 \%{ }^{25,29,3033-36,40}$ The mean age of development of AIHA was 13.7-17.5 (1-58) months in two studies (Table 3) ${ }^{29,33}$ All patients had AIHA onset aged $<5$ years. ${ }^{33}$ The commonest clinical presentations of AIHA include anemia, jaundice, and hepatosplenomegaly. ${ }^{29,40}$ Positive antinuclear antibodies have been demonstrated in $5 \%-9 \%$ of patients with AIHA, while Coombs tests were positive in some patients. ${ }^{29,40}$ 


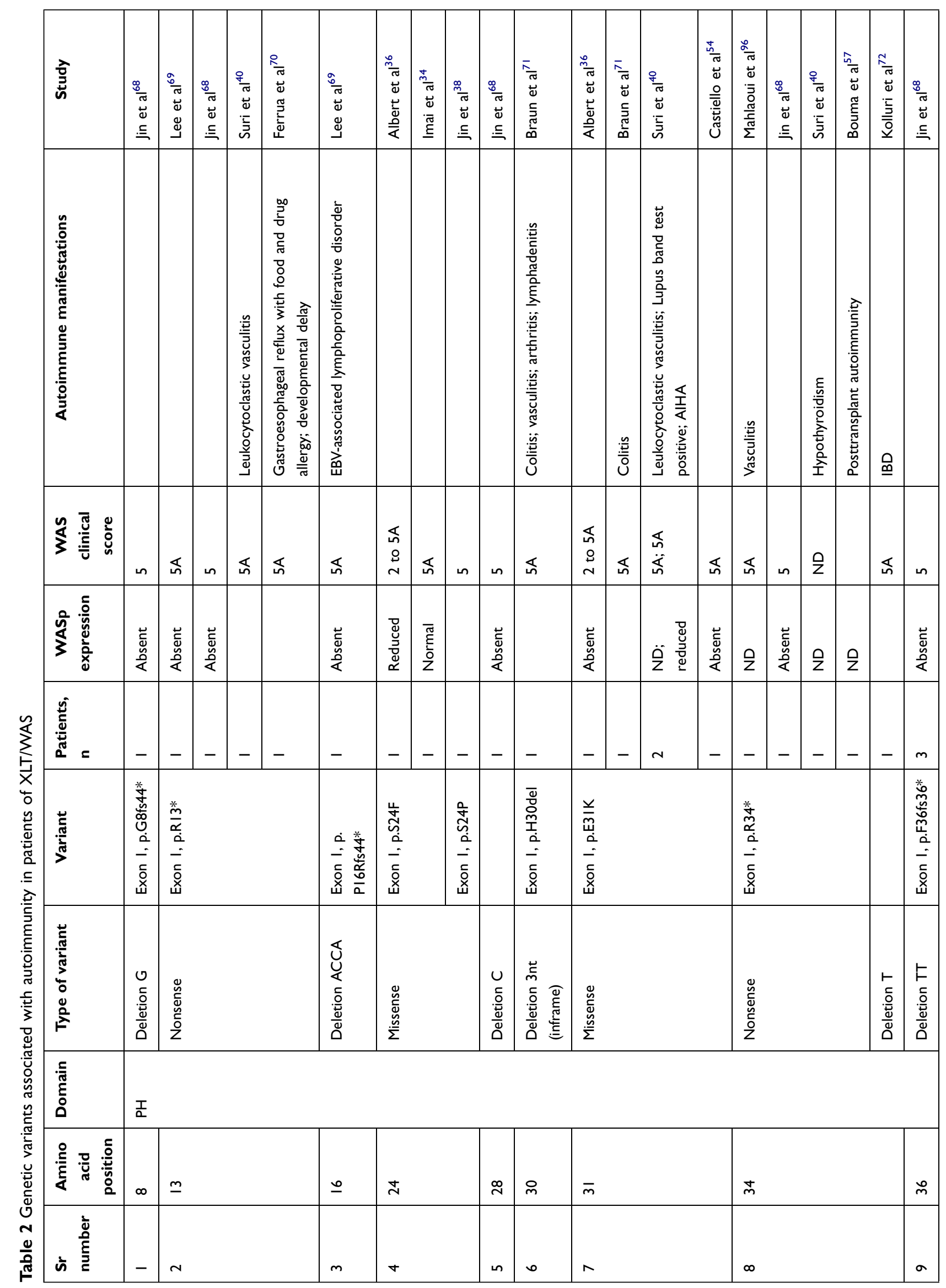




\begin{tabular}{|c|c|c|c|c|c|c|c|c|c|c|c|c|c|c|c|c|c|}
\hline 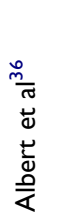 & 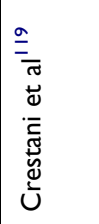 & 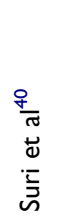 & 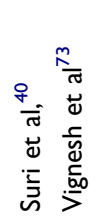 & 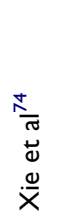 & $\begin{array}{l}\frac{\infty}{\sigma} \\
\stackrel{\Xi}{0} \\
\stackrel{\Xi}{\cong}\end{array}$ & 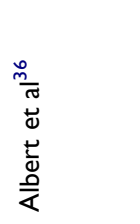 & 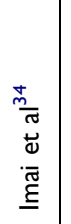 & 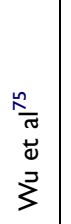 & 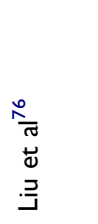 & 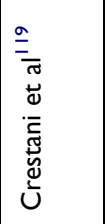 & 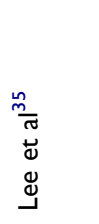 & 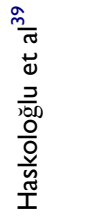 & 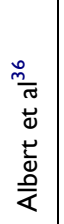 & 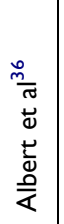 & 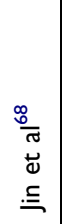 & 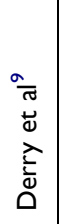 & 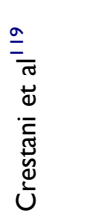 \\
\hline & 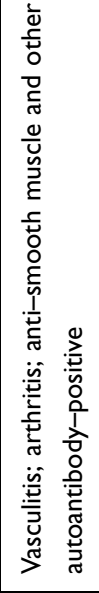 & 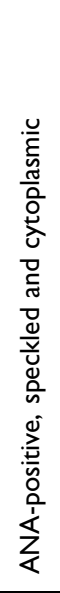 & 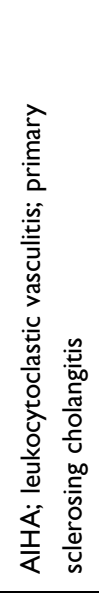 & 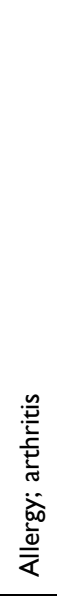 & & & & & & 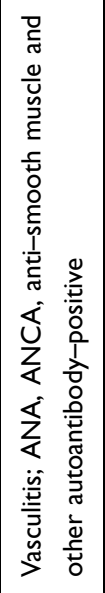 & 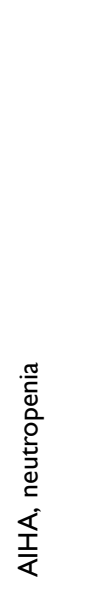 & 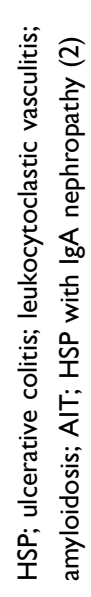 & & & & & 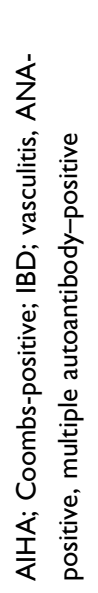 \\
\hline 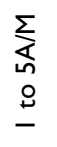 & 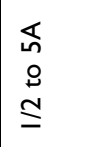 & $\mathbb{\pi}$ & ธ్ & ஸ็ & in & 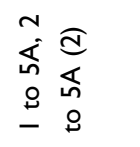 & in & 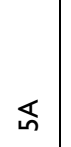 & 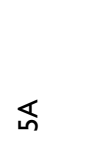 & $\begin{array}{l}\mathbb{1} \\
0 \\
0 \\
\text { I }\end{array}$ & 芯 & 迥 & $\begin{array}{l}\mathbb{1} \\
0 \\
\stackrel{1}{-} \\
-1\end{array}$ & 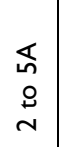 & in & ธ్ & in \\
\hline 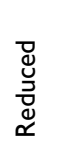 & & 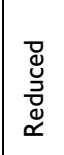 & 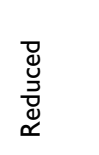 & & 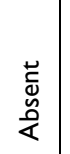 & 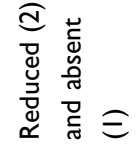 & $\begin{array}{l}\overline{\widetilde{\Xi}} \\
\stackrel{\bar{g}}{\mathrm{O}} \\
\mathrm{Z}\end{array}$ & 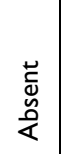 & 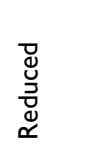 & & & & 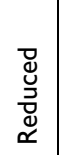 & 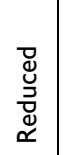 & १ & 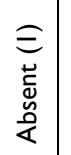 & \\
\hline- & - & - & - & - & - & $m$ & - & - & - & - & - & in & - & - & - & $N$ & - \\
\hline 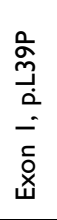 & & & 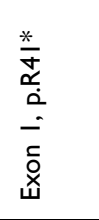 & & & 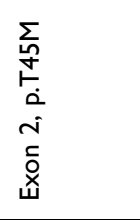 & & & 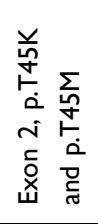 & 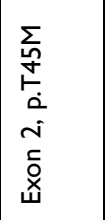 & 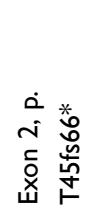 & 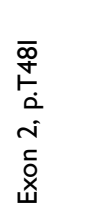 & 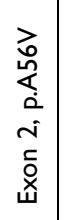 & 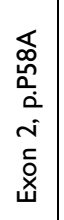 & 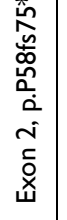 & & 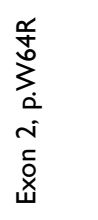 \\
\hline 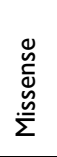 & & & 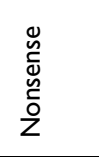 & & & 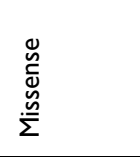 & & & & & 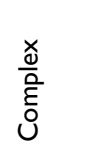 & 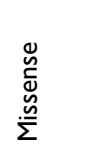 & 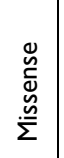 & 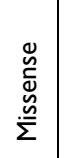 & 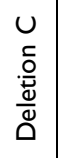 & 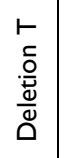 & 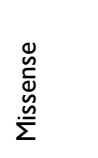 \\
\hline \multicolumn{18}{|c|}{$\begin{array}{l}\text { 巳 } \\
\text { J } \\
\text { I } \\
\frac{I}{\alpha} \\
\end{array}$} \\
\hline 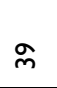 & & & $\bar{\gamma}$ & & & ケ & & & & & & $\stackrel{\infty}{+}$ & セ̊ & $\stackrel{\infty}{\circledR}$ & & 8 & ఫర \\
\hline 으 & & & $\simeq$ & & & $\underline{m}$ & & & & & & \pm & $\underline{\underline{ }}$ & $\underline{0}$ & & $\simeq$ & $\underline{\infty}$ \\
\hline
\end{tabular}




\begin{tabular}{|c|c|c|c|c|c|c|c|c|c|c|c|c|c|c|c|c|}
\hline $\begin{array}{l}\text { ते } \\
\text { ज्ञ }\end{array}$ & 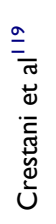 & 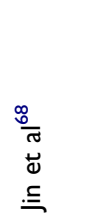 & 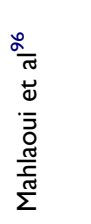 & 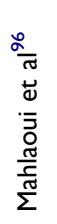 & 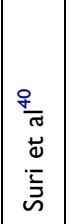 & 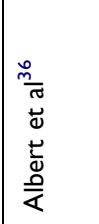 & 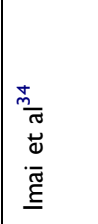 & 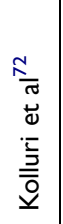 & 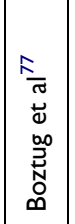 & 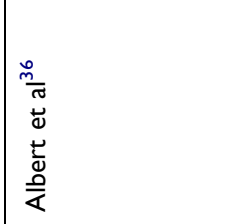 & 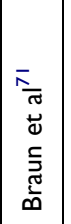 & 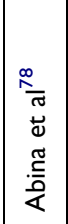 & 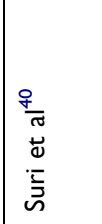 & 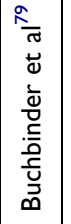 & $\mid \begin{array}{l}\frac{0}{5} \\
\frac{\pi}{0} \\
\frac{\pi}{0} \\
\frac{0}{0} \\
0\end{array}$ & 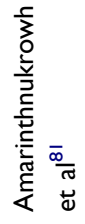 \\
\hline 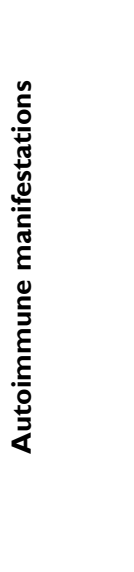 & $\underline{\underline{Q}}$ & & 午 & $\underset{\frac{1}{\tau}}{\mathbb{r}}$ & 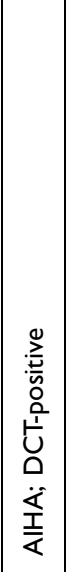 & & & 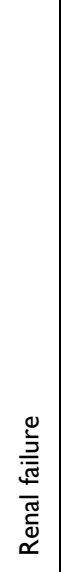 & 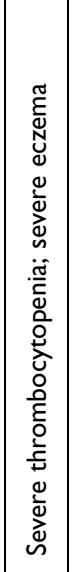 & & 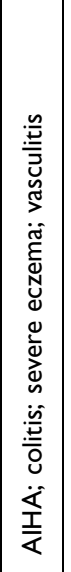 & $\stackrel{上}{\varangle}$ & 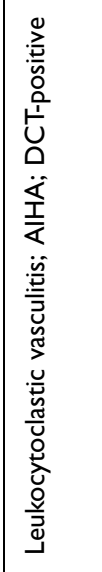 & $\frac{5}{<}$ & & \\
\hline 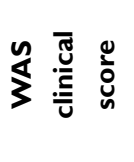 & 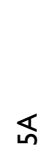 & in & ก็ & 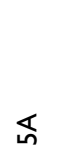 & ถึ & 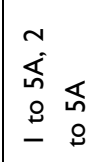 & $\sum_{i}^{\infty}$ & in & $\mathbb{\pi}$ & 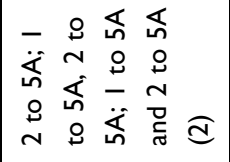 & ถึ & 吕 & $\begin{array}{l}\text { 昏 } \\
\text { î́ }\end{array}$ & ஸ็ & ஸा & ஸ็ \\
\hline 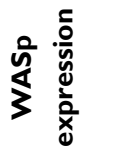 & $\mathrm{Q}$ & $\begin{array}{l}\text { 岕 } \\
\text { 䐱 }\end{array}$ & $\begin{array}{l}\text { 密 } \\
\text { 产 }\end{array}$ & $\mathrm{Q}$ & 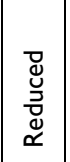 & 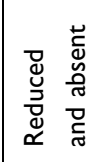 & 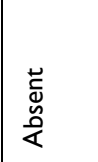 & & & 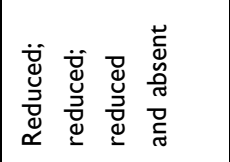 & & \begin{tabular}{|l|}
$\bar{\sigma}$ \\
$\tilde{z}$ \\
$\bar{z}$
\end{tabular} & 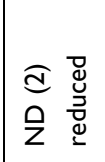 & & & \\
\hline 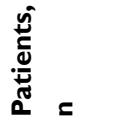 & - & - & - & - & - & $N$ & - & - & - & $\begin{array}{l}m \\
\ddot{\overline{-}}\end{array}$ & - & - & $m$ & - & - & - \\
\hline 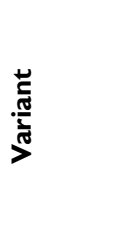 & 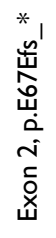 & 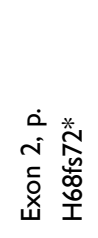 & 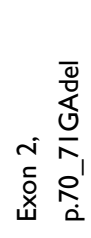 & 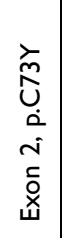 & 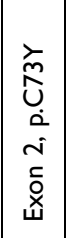 & 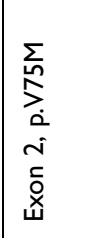 & 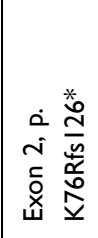 & 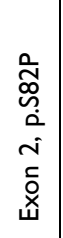 & 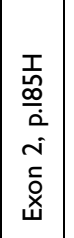 & 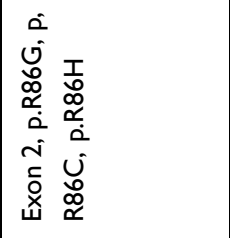 & 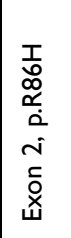 & & & $\begin{array}{l}u \\
o \\
\infty \\
\alpha \\
\dot{\alpha} \\
i \\
i \\
\dot{0} \\
\dot{x}\end{array}$ & & 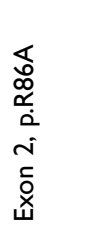 \\
\hline 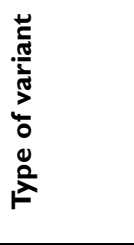 & 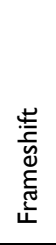 & 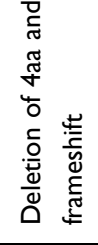 & 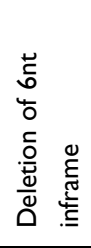 & 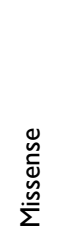 & & 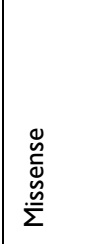 & 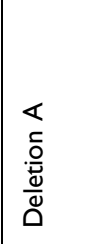 & 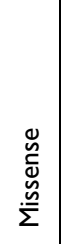 & 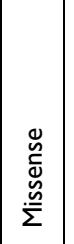 & 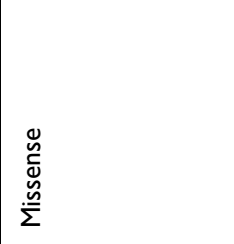 & & & & & & \\
\hline 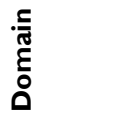 & & & & & & & & & & & & & & & & \\
\hline 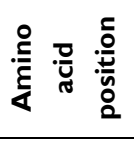 & $\hat{0}$ & థ & $\stackrel{R}{R}$ & $\stackrel{n}{\wedge}$ & & $\stackrel{n}{n}$ & $\stackrel{0}{1}$ & $\infty$ & œ & $\infty$ & & & & & & \\
\hline iे & $\underline{a}$ & i & $\bar{N}$ & $\pi$ & & $\ddot{\sim}$ & \pm & $\stackrel{\stackrel{2}{\sim}}{2}$ & జి & $\lesssim$ & & & & & & \\
\hline
\end{tabular}




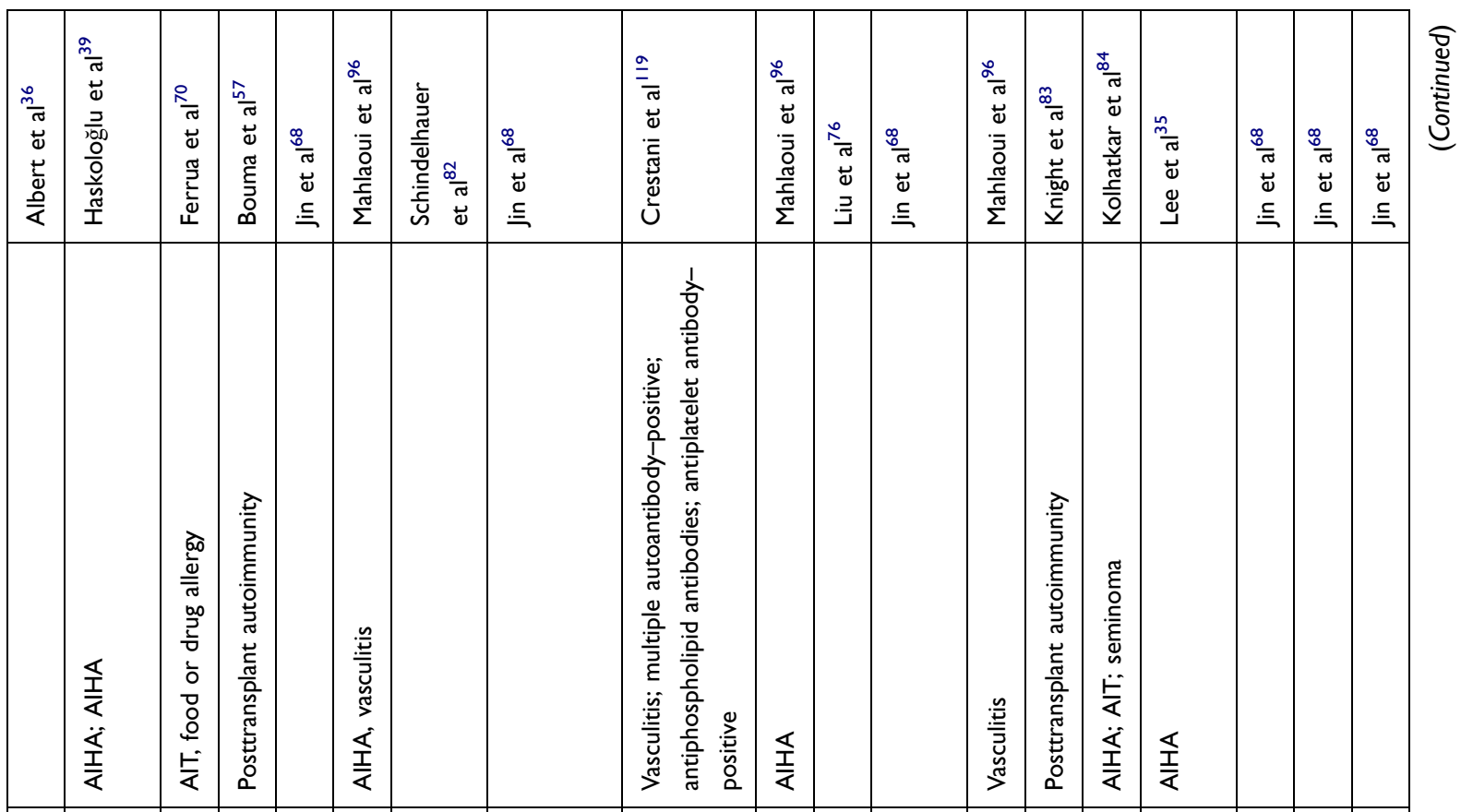

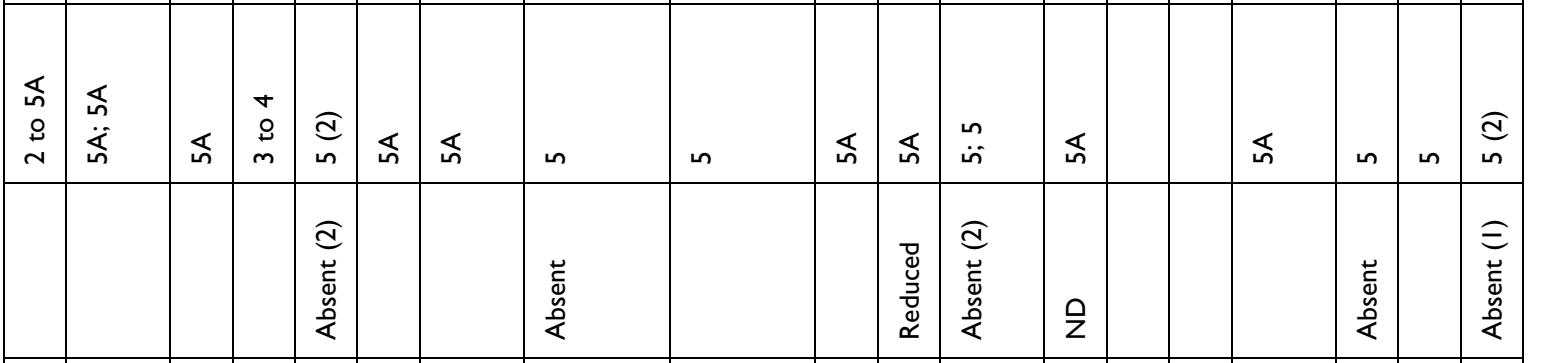

\begin{tabular}{|c|c|c|c|c|c|c|c|c|c|c|c|c|c|c|c|c|c|c|}
\hline- & $\ddot{-}$ & - & - & $N$ & - & - & - & - & - & - & $N$ & - & - & & - & - & - & $N$ \\
\hline 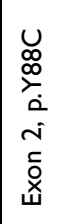 & 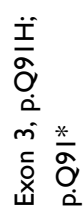 & 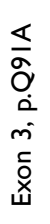 & 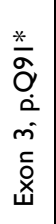 & 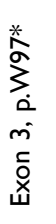 & & 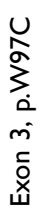 & 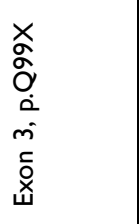 & 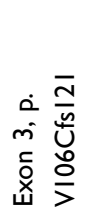 & 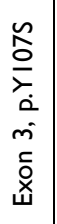 & $\begin{array}{l}u \\
\hat{0} \\
\frac{0}{i} \\
\dot{0} \\
\dot{m} \\
\overline{0} \\
\dot{0} \\
\dot{x}\end{array}$ & 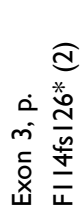 & 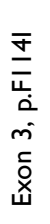 & 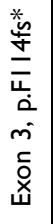 & 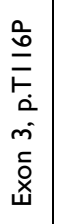 & 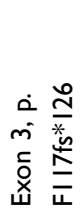 & 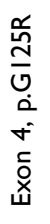 & 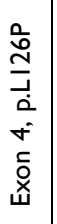 & 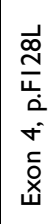 \\
\hline 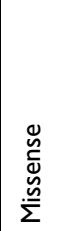 & 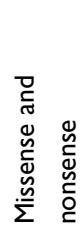 & 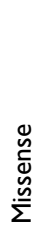 & 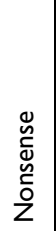 & 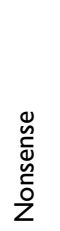 & & 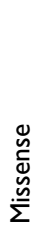 & 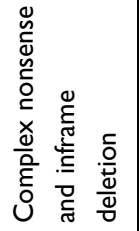 & 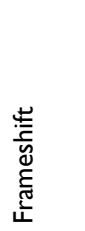 & 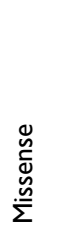 & & 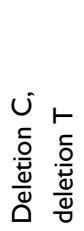 & 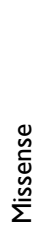 & 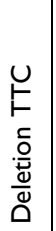 & 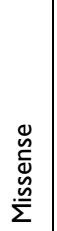 & o. & 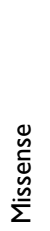 & 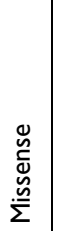 & ֻू \\
\hline
\end{tabular}

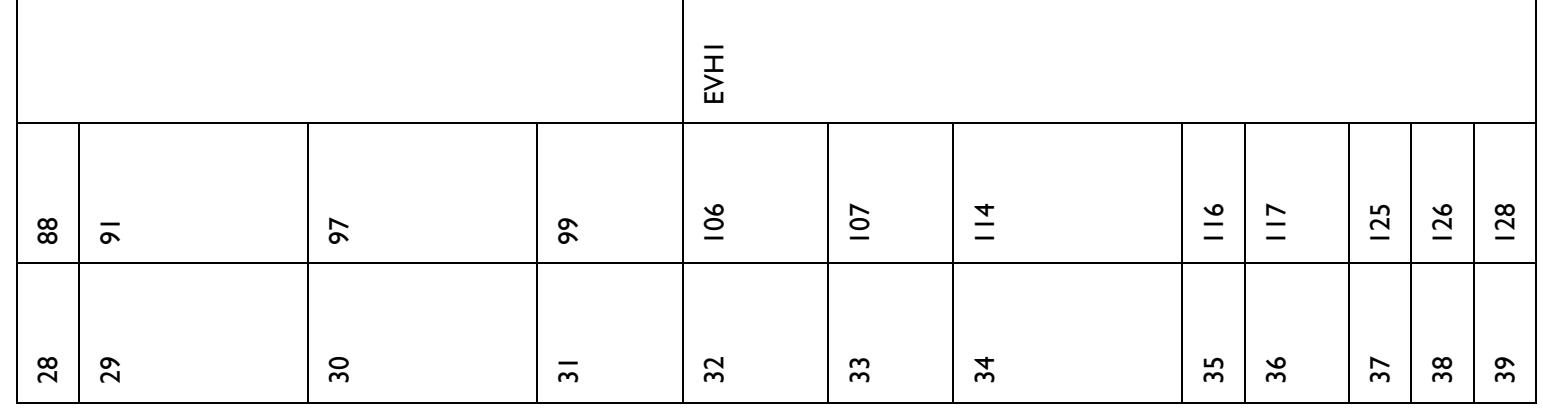




\begin{tabular}{|c|c|c|c|c|c|c|c|c|c|c|c|c|c|c|c|c|}
\hline $\begin{array}{l}\text { ते } \\
\overrightarrow{\tilde{n}}\end{array}$ & 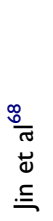 & 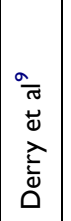 & 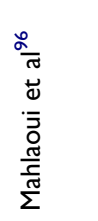 & 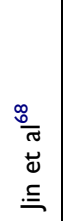 & 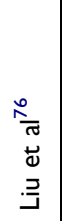 & 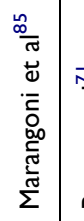 & 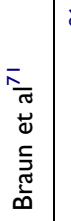 & 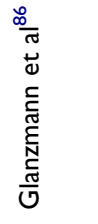 & 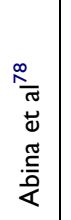 & 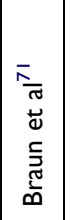 & $\begin{array}{l}\frac{\infty}{\pi} \\
\stackrel{\Xi}{ \pm} \\
\cong\end{array}$ & 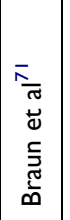 & $\begin{array}{l}\frac{\infty}{\sigma} \\
\stackrel{0}{ \pm} \\
\cong\end{array}$ & 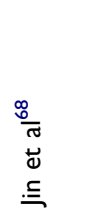 & 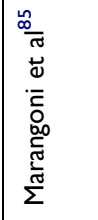 & 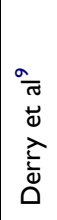 \\
\hline 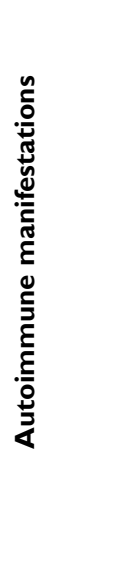 & & & 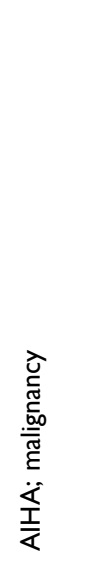 & & & 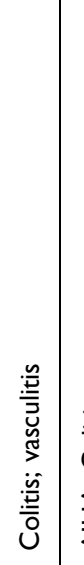 & 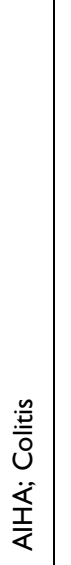 & 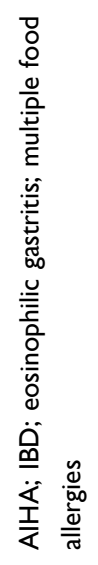 & 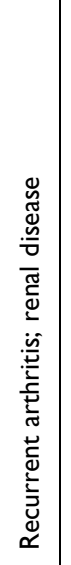 & $\frac{a}{\overline{\frac{E}{0}}}$ & & $\frac{\stackrel{n}{\underline{E}}}{0}$ & & & 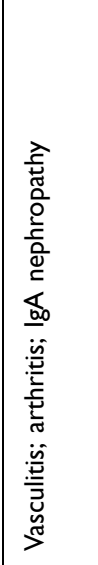 & \\
\hline 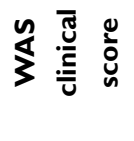 & in & เీ & $\sum_{i}^{\sum}$ & in & ถీ & กீ & 氐 & ถึ & ถึ & เீ & in & ถึ & $\underset{\mathrm{n}}{\widehat{m}}$ & in & เึ & 吕 \\
\hline 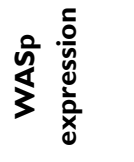 & 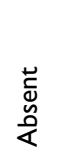 & & 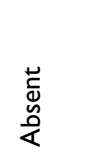 & $\mathrm{Q}$ & 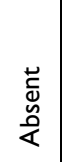 & & & & 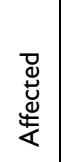 & & 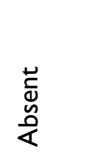 & & 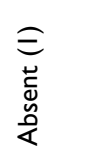 & & & \\
\hline 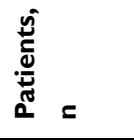 & - & - & - & - & - & - & - & & - & - & - & - & $m$ & - & - & - \\
\hline 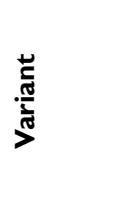 & 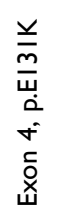 & 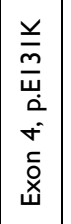 & 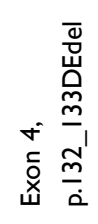 & 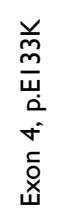 & & & & & 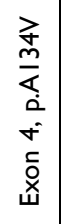 & 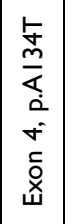 & 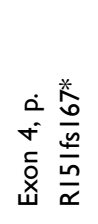 & 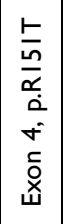 & 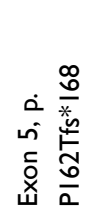 & 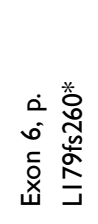 & 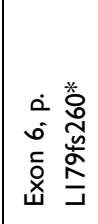 & 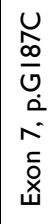 \\
\hline 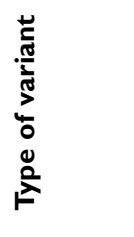 & 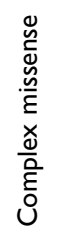 & 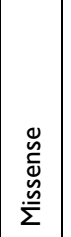 & 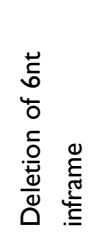 & 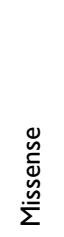 & & & & & 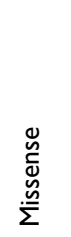 & & 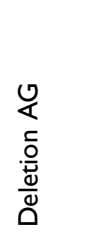 & 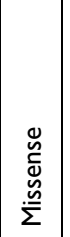 & 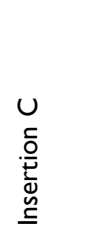 & 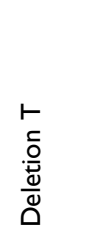 & $\begin{array}{l}\vdash \\
\bar{o} \\
\frac{.0}{\tilde{D}} \\
\Delta\end{array}$ & 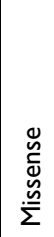 \\
\hline 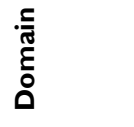 & & & & & & & & & & & & & $\stackrel{m}{\underline{m}}$ & & & \\
\hline 总 & $\overline{\underline{m}}$ & & $\tilde{\check{n}}$ & $\underline{\underline{m}}$ & & & & & $\underline{\underline{m}}$ & & 뜨 & & $\underline{\sigma}$ & $\underline{\Omega}$ & & $\underline{\underline{\omega}}$ \\
\hline जे & q & & $\bar{\gamma}$ & $\mathcal{F}$ & & & & & $\stackrel{m}{\sim}$ & & J & & 年 & fo & & $\hat{f}$ \\
\hline
\end{tabular}




\begin{tabular}{|c|c|c|c|c|c|c|c|c|c|c|c|c|c|}
\hline 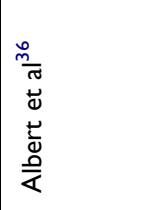 & 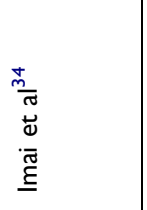 & 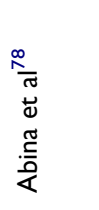 & 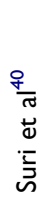 & $\begin{array}{l}\frac{\infty}{\sigma} \\
\stackrel{0}{0} \\
\stackrel{\Xi}{\Xi}\end{array}$ & 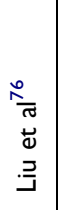 & 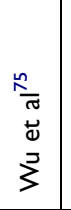 & 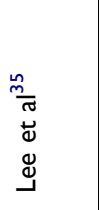 & 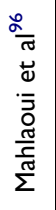 & $\begin{array}{l}\frac{\infty}{\pi} \\
\stackrel{\varpi}{ \pm} \\
\stackrel{\equiv}{=}\end{array}$ & 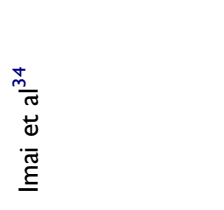 & 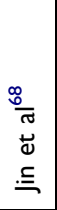 & 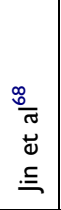 & 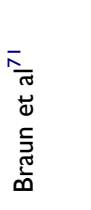 \\
\hline & & 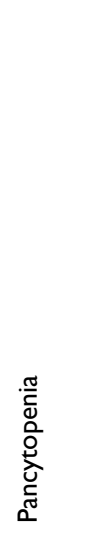 & 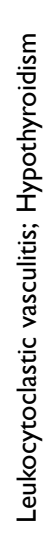 & & & & $\begin{array}{l}\widehat{d} \\
\frac{1}{1} \\
\frac{1}{4}\end{array}$ & 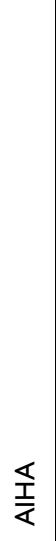 & & & & & 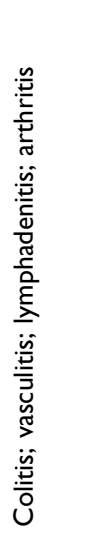 \\
\hline $\begin{array}{l}a \\
\mathbb{a} \\
0 \\
\dot{2} \\
N \\
\end{array}$ & 氐 & 通 & ถึ & in & 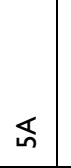 & ถึ & $\begin{array}{l}\widehat{d} \\
\text { sin }\end{array}$ & 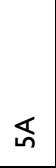 & in & 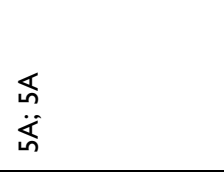 & in & in & 绾 \\
\hline 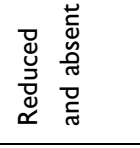 & $\begin{array}{l}\overline{\widetilde{g}} \\
\bar{\xi} \\
\text { zे } \\
\end{array}$ & $\begin{array}{l}\overline{\widetilde{g}} \\
\bar{\xi} \\
\text { ż } \\
\end{array}$ & 乙 & 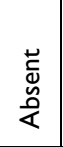 & 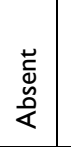 & 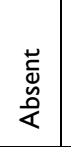 & & 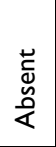 & 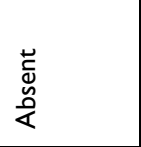 & 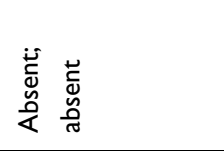 & & 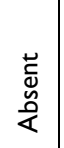 & \\
\hline N & - & - & - & - & - & - & $N$ & - & & & - & - & - \\
\hline 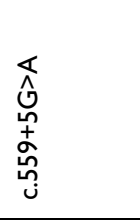 & 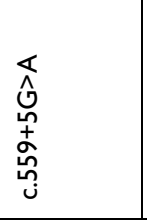 & 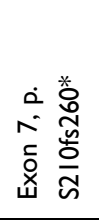 & 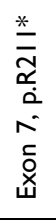 & & & & 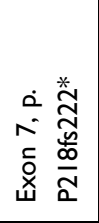 & 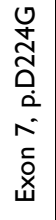 & $*$ & * & 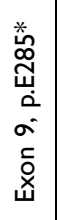 & 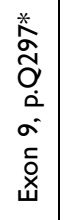 & 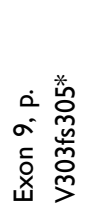 \\
\hline 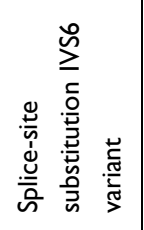 & 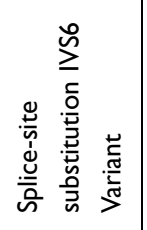 & 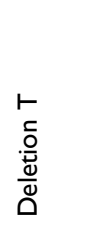 & 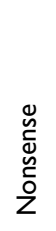 & & & & 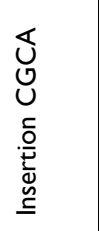 & 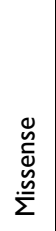 & 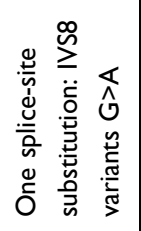 & 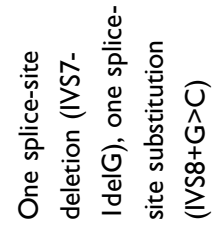 & 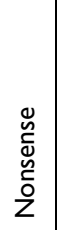 & 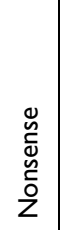 & 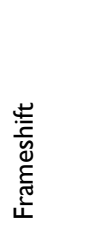 \\
\hline & & & & & & & & $\infty$ & \multicolumn{3}{|l|}{$\stackrel{\varrho}{\underline{x}}$} & 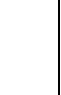 & \\
\hline \& & & $\stackrel{\circ}{N}$ & $\overline{\bar{N}}$ & & & & $\frac{\infty}{N}$ & $\underset{\sim}{ \pm}$ & $\stackrel{P}{\sim}$ & & $\stackrel{\leftrightarrow \infty}{\sim}$ & $\widehat{\grave{N}}$ & 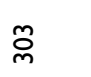 \\
\hline$\stackrel{\infty}{+}$ & & $\underset{\sigma}{q}$ & 요 & & & & in & กี & กี & & 亡 & 吅 & เீ \\
\hline
\end{tabular}




\begin{tabular}{|c|c|c|c|c|c|c|c|c|c|c|c|c|c|c|c|c|c|}
\hline 㝘 & $\begin{array}{c}\frac{\infty}{\pi} \\
\stackrel{0}{0} \\
\stackrel{\Xi}{\Xi} \\
\end{array}$ & 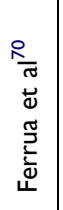 & 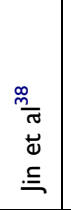 & 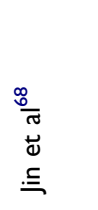 & 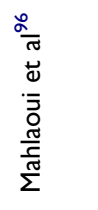 & 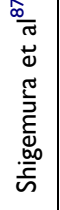 & 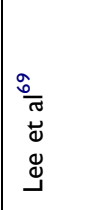 & $\begin{array}{l}\frac{\infty}{\pi} \\
\stackrel{0}{0} \\
\stackrel{\Xi}{\Xi}\end{array}$ & 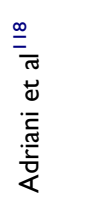 & $\begin{array}{l}\frac{\infty}{\pi} \\
\stackrel{0}{0} \\
\stackrel{\Xi}{\Xi}\end{array}$ & 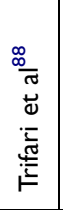 & $\begin{array}{l}\frac{\infty}{\pi} \\
\stackrel{0}{0} \\
\stackrel{\Xi}{\Xi}\end{array}$ & 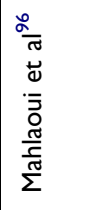 & 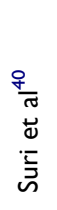 & 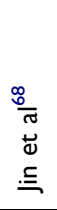 & 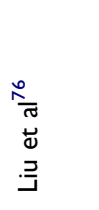 & 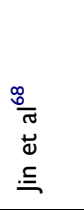 \\
\hline 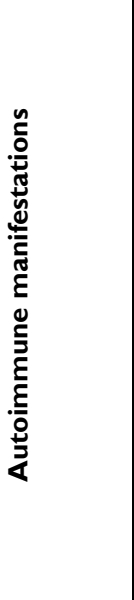 & & 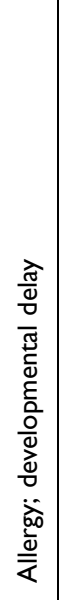 & & & $\begin{array}{l}\widehat{a} \\
\mathbb{x} \\
\frac{1}{\alpha}\end{array}$ & 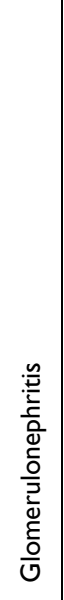 & 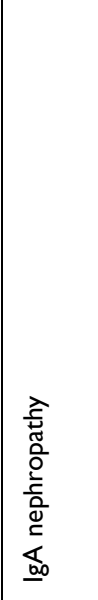 & & 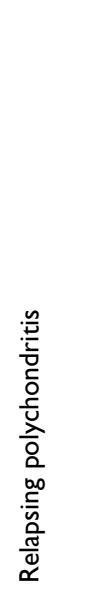 & & 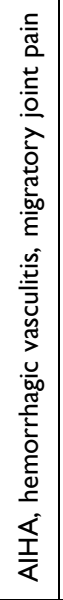 & & & 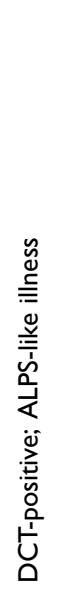 & & & \\
\hline 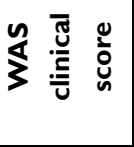 & $\begin{array}{c}\widehat{m} \\
n\end{array}$ & 芯 & in & in & $\begin{array}{l}\widehat{a} \\
\mathbb{s} \\
\text { in }\end{array}$ & « & $\begin{array}{l}\text { 占 } \\
\text { d } \\
\text { v }\end{array}$ & in & เn & in & 吕 & in & 氐 & 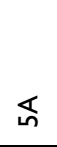 & in & 氐 & in \\
\hline 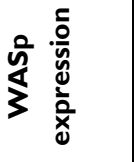 & 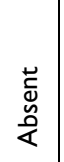 & & & 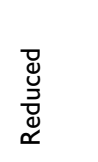 & $\mathrm{Q}$ & & 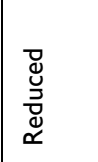 & 之 & 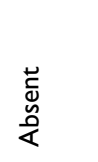 & 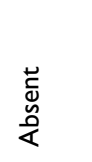 & & & 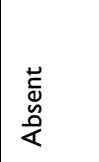 & 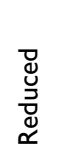 & & 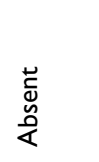 & 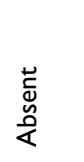 \\
\hline 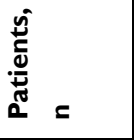 & $m$ & - & - & - & r & - & - & - & - & - & - & - & - & - & - & - & - \\
\hline 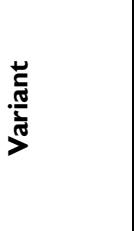 & 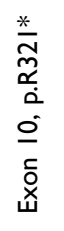 & & & 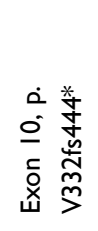 & 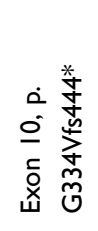 & 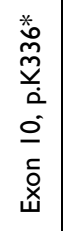 & 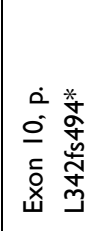 & 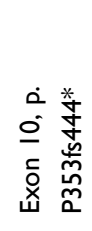 & 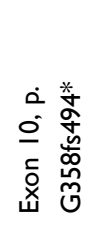 & 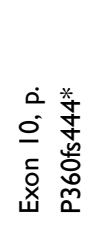 & 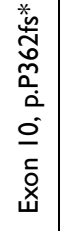 & 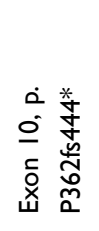 & 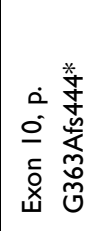 & 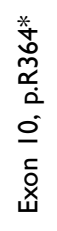 & & 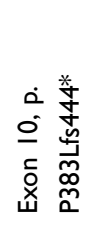 & 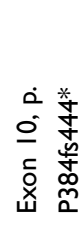 \\
\hline 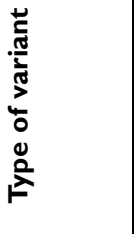 & 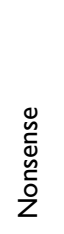 & & & 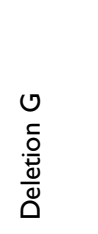 & 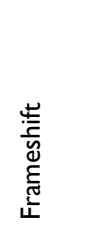 & 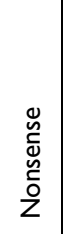 & 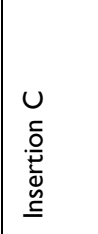 & 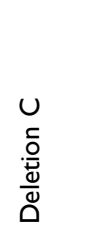 & 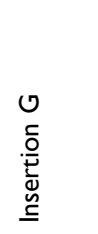 & 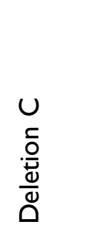 & 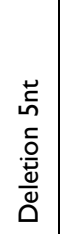 & 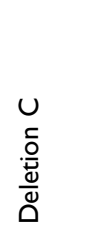 & 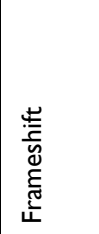 & 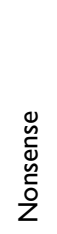 & & 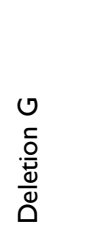 & 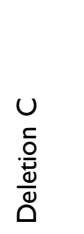 \\
\hline هั & $\stackrel{\frac{\alpha}{\alpha}}{\alpha}$ & & & & & 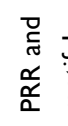 & & 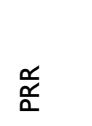 & & & & & & & & 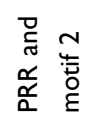 & \\
\hline 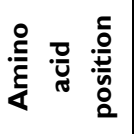 & $\overline{\widetilde{m}}$ & & & $\stackrel{\widetilde{m}}{\tilde{m}}$ & 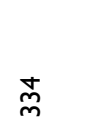 & ల్లి & f & $\stackrel{\mathscr{n}}{m}$ & 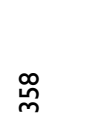 & : & స్ల & & 芯 & 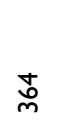 & & $\underset{m}{\infty}$ & 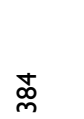 \\
\hline ทे & กิ & & & $\stackrel{\infty}{\circ}$ & ติ & 8 & $\overline{0}$ & $\tilde{\sigma}$ & $\tilde{6}$ & కt & น & & ๑ & $\hat{0}$ & & ల్ర & a \\
\hline
\end{tabular}


Dovepress

Sudhakar et al

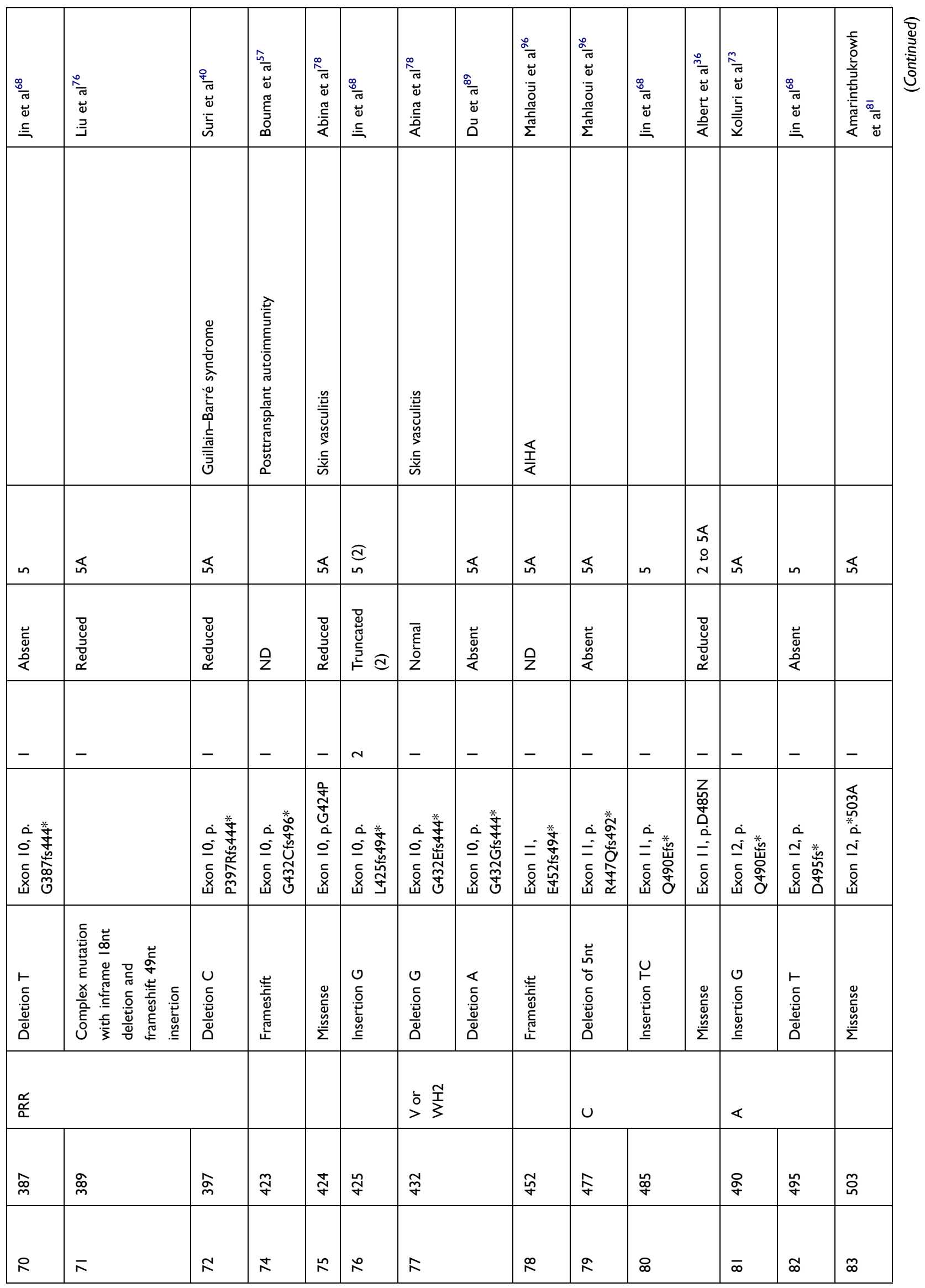

The Application of Clinical Genetics 2021:14

https://doi.org// 0.21 47/TACG.S213920
DovePress 


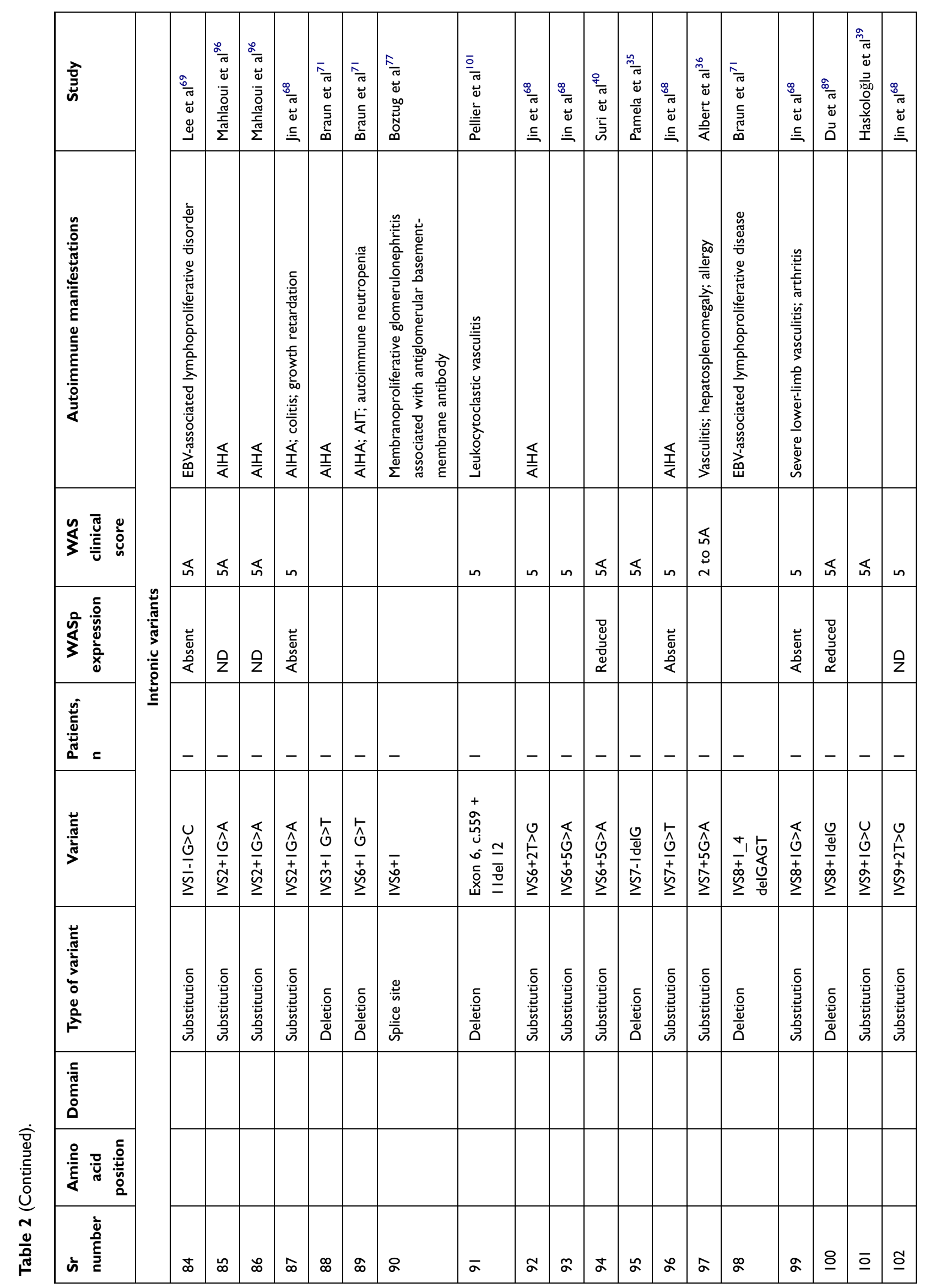




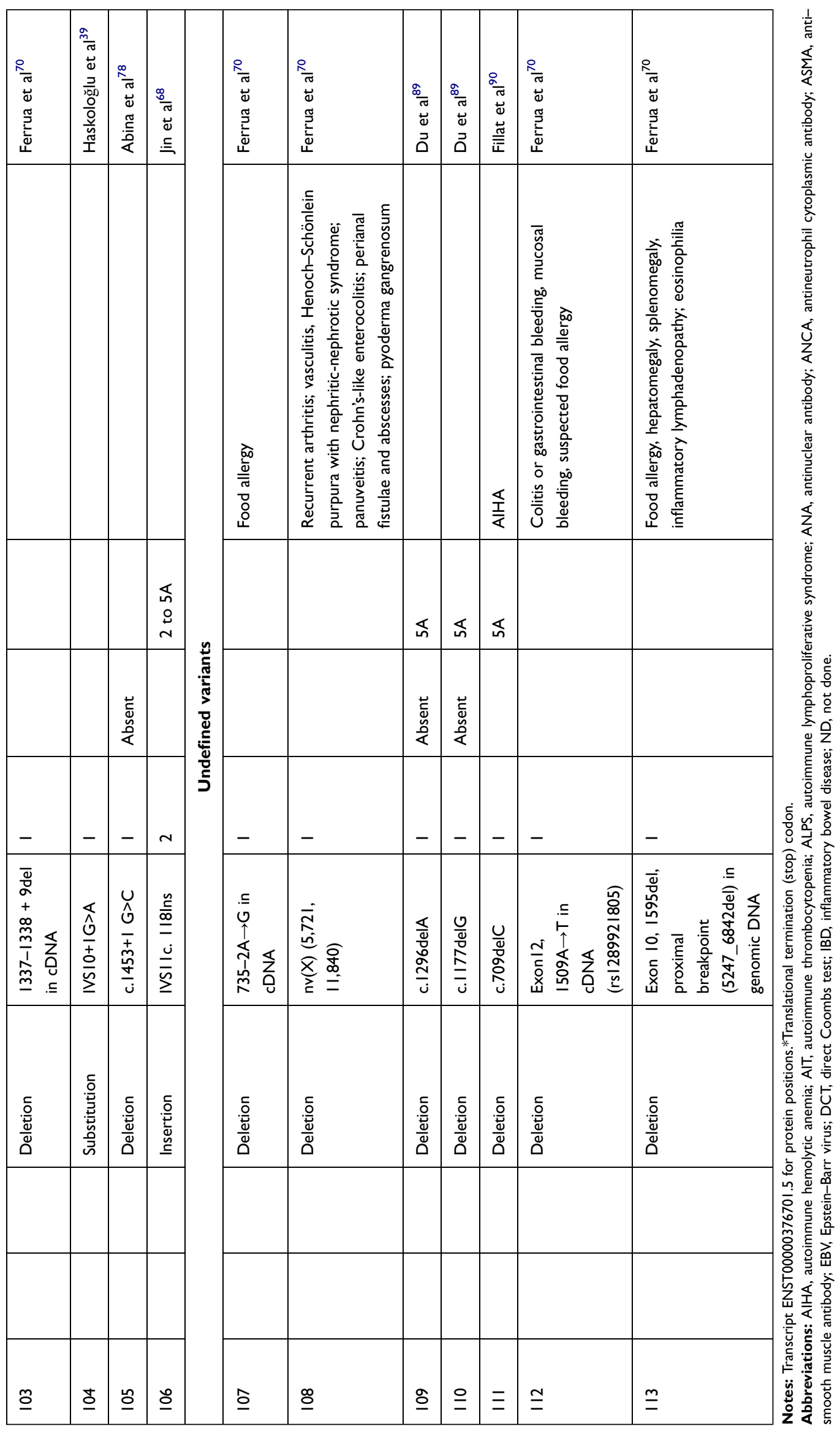




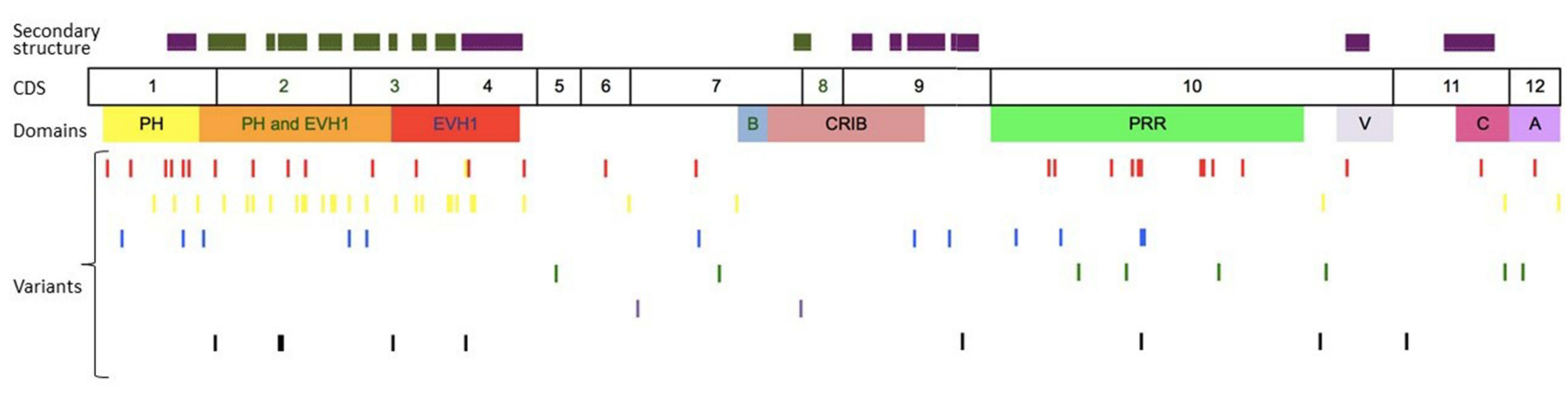

Figure 2 Variants reported in patients of XLT/WAS with autoimmunity. Secondary structure of WASp: $\alpha$-helix (purple) and $\beta$-strands (green)., CDS, coding sequence; PH, pleckstrin homology; EVHI, vasodilator-stimulated phosphoprotein homology; B, basic; CRIB, Cdc42-and Rac-interactive binding; PRR, proline-rich region; V, verprolin; C, central; A, acidic; . Reported variants: deletions (red), missense (yellow), nonsense (blue), insertions (green), splice site (pink), complex and undefined frameshift variants (black).

\section{Autoimmune Thrombocytopenia (AIT)}

Microthrombocytopenia is a cardinal feature of WAS. Abnormality in WASp results in megakaryocyte dysfunction, leading to formation of small platelets. These abnormal microplatelets are recognized by self-antibodies and are prematurely cleared by the spleen. ${ }^{91-93}$ Immunomediated thrombocytopenia also plays a contributing role in $15 \%-32 \%$ of patients (Tables 1 and 2). ${ }^{29,30,33,36}$ Antiplatelet antibodies have been found in $40 \%$ of WASp-deficient mice. ${ }^{94}$ Assays for antiplatelet antibodies are difficult to standardize and may not be easily available, especially in developing countries. Moreover, these antibodies may have a poor correlation with AIT. ${ }^{95}$ AIT may evolve over and exacerbate the underlying thrombocytopenia that is characteristic of WAS. A sudden drop in baseline platelet counts (usually $<10 \times 10^{9} / \mathrm{L}$ ) with or without overt clinical bleeding is an important clinical clue to emergence of AIT. Failure to demonstrate a significant rise or fall in platelet count after platelet transfusion may herald the development of AIT. ${ }^{95}$ Most autoimmune manifestations evolve over time, but immunothrombocytopenia may have an early age of onset. In a cohort of patients with WAS aged $<2$ years with a clinical severity score of 5 , ten of $26(38.4 \%)$ had antiplatelet antibody-positive severe refractory thrombocytopenia. ${ }^{96}$ Intracranial hemorrhage is a major cause of mortality in patients with WAS. ${ }^{25}$

Differentiating AIT from baseline thrombocytopenia in WAS is crucial, as management strategies vary. Institution of appropriate immunosuppressive therapy is needed to maintain platelet counts.

\section{Autoimmune Neutropenia}

Autoimmune neutropenia is found in $2 \%-25 \%$ of patients with WAS. $^{25,29,30,33,35}$

\section{Vasculitides}

Vasculitis is the second-commonest autoimmune manifestation, and has been found in $1.5 \%-29 \%$ of patients with WAS. It accounts for $6 \%-45 \%$ of all autoimmune manifestations. ${ }^{25,30,33,34,36,39,40}$ (Tables 1 and 2)

Two patterns of vasculitic abnormality have been reported in WAS: medium-sized and small-vessel vasculitis of skin, renal, coronary, cerebral, or hepatic arteries, and large-vessel vasculitis involving the aorta and its major branches. ${ }^{40,5097-101}$ Involvement of small vessels, especially those of the skin, ${ }^{25,33,39,102,103}$ is the commonest vasculitic abnormality (75\%). ${ }^{33}$ IgA vasculitis (previously termed Henoch-Schönlein purpura) has been reported in $28.5 \%$ of patients with vasculitis. ${ }^{25}$ Kawakami et al described Kawasaki disease in a patient with WAS. ${ }^{104}$ Involvement of small- and medium-sized arteries of the gastrointestinal tract, ${ }^{97,105,106}$ heart, ${ }^{104,105}$ liver, ${ }^{98,104}$ gallbladder, ${ }^{105}$ kidneys, ${ }^{98,106}$ stomach,${ }^{102}$ and cerebral blood vessels, ${ }^{98,107}$ has also been reported. In a singlecenter study of 55 patients of WAS, Dupuis-Girod et al noted cutaneous vasculitis at a mean age of 52.5 (11-184) months. ${ }^{33}$ Lao et al reported large-vessel vasculitis involving the aorta and renal artery in a 5-year-old boy with WAS. ${ }^{100}$ Pellier et al described five children with WAS who developed aortic aneurysms predominantly involving the thoracic and abdominal aortae. ${ }^{101}$ Four of these five patients with vasculitis were asymptomatic, and aneurysms were discovered only on screening.

Predisposition to developing vasculitis has been ascribed to immunodysregulation in WAS. Patients with WAS typically have depressed levels of IgM and elevated levels of IgA and IgE. It has been suggested that immunodeposition within the vessel wall can lead to necrotizing vasculitis. ${ }^{98}$ Alternatively, vasculitis could result from an infectious insult due to the underlying immunodeficiency. 


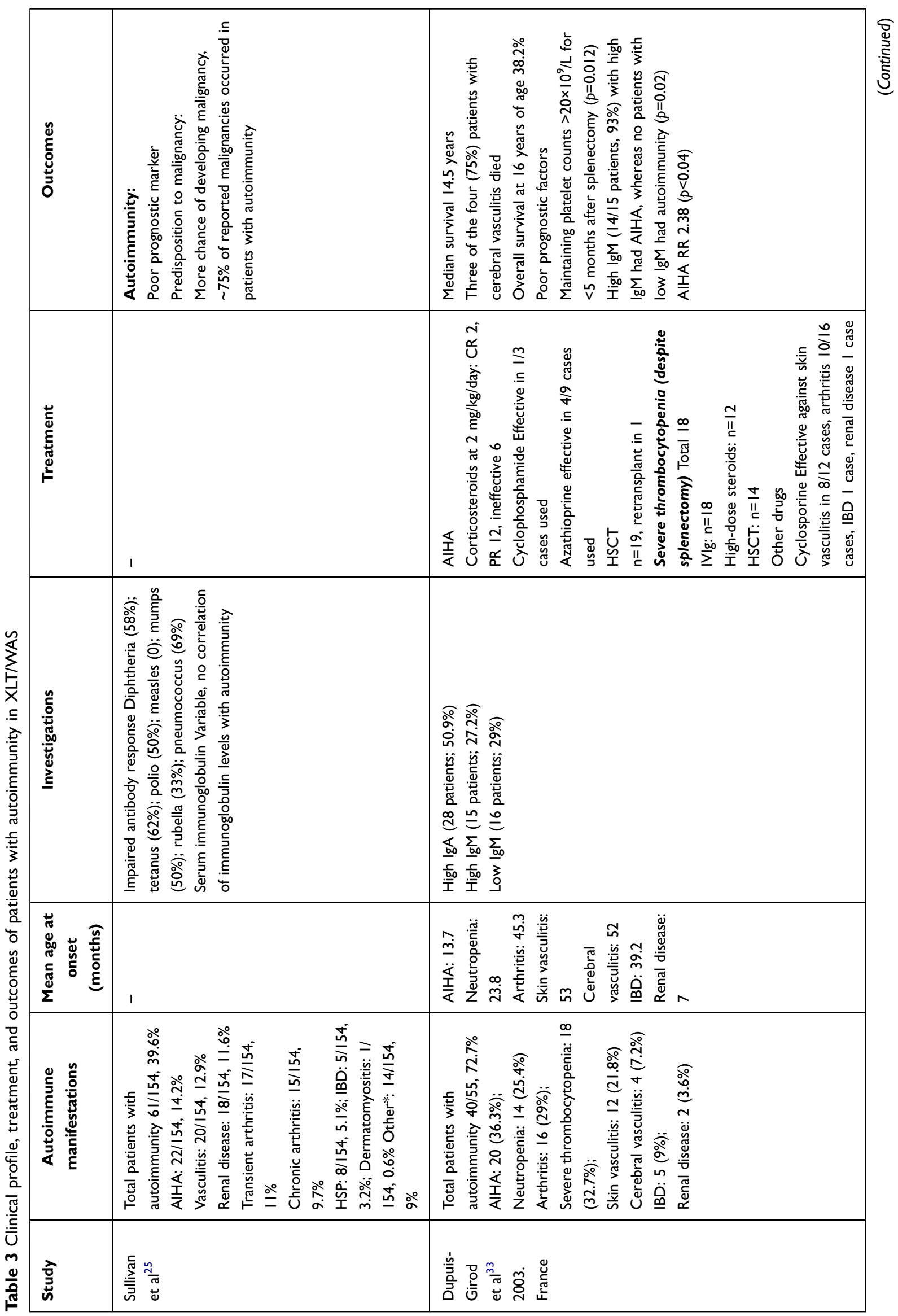




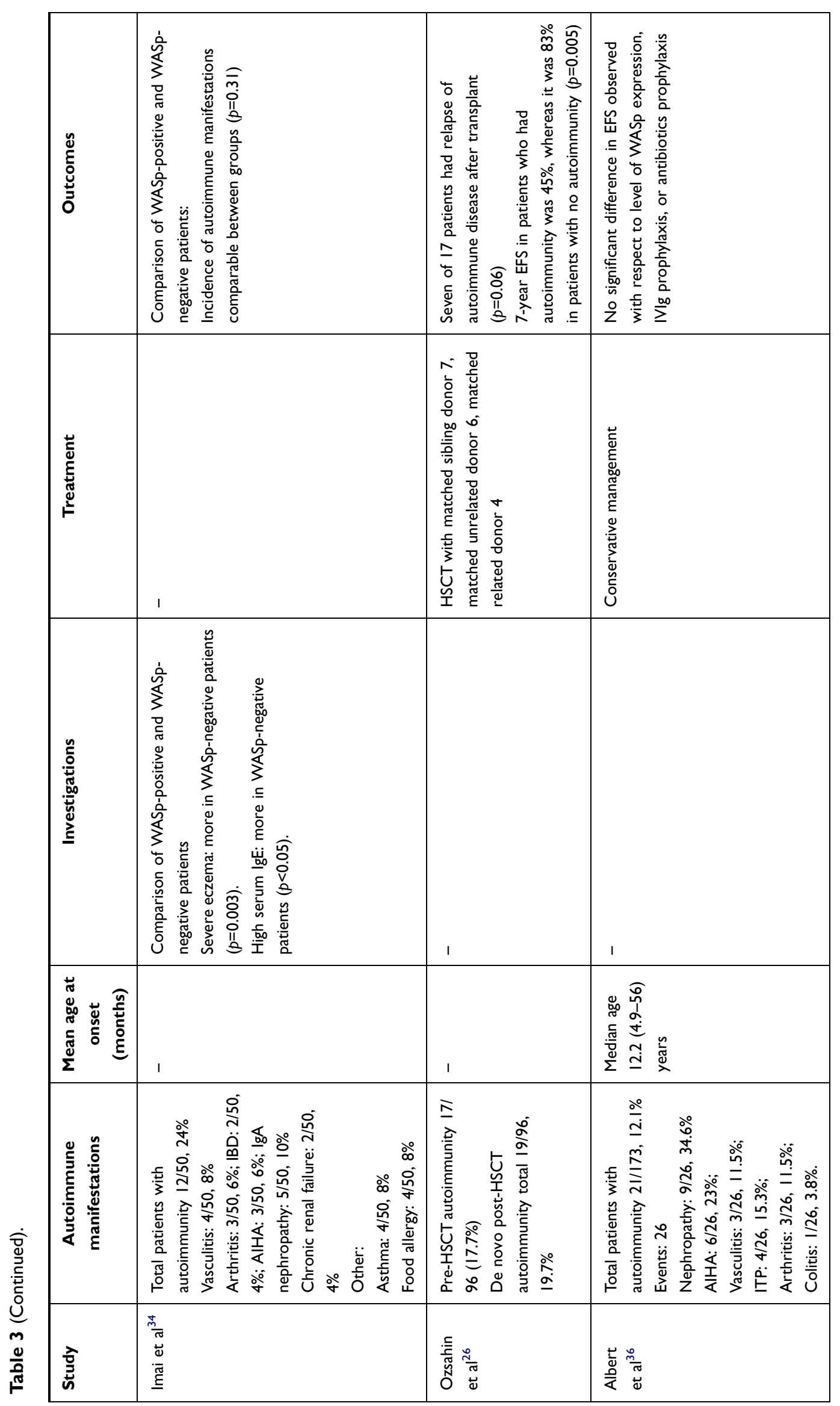




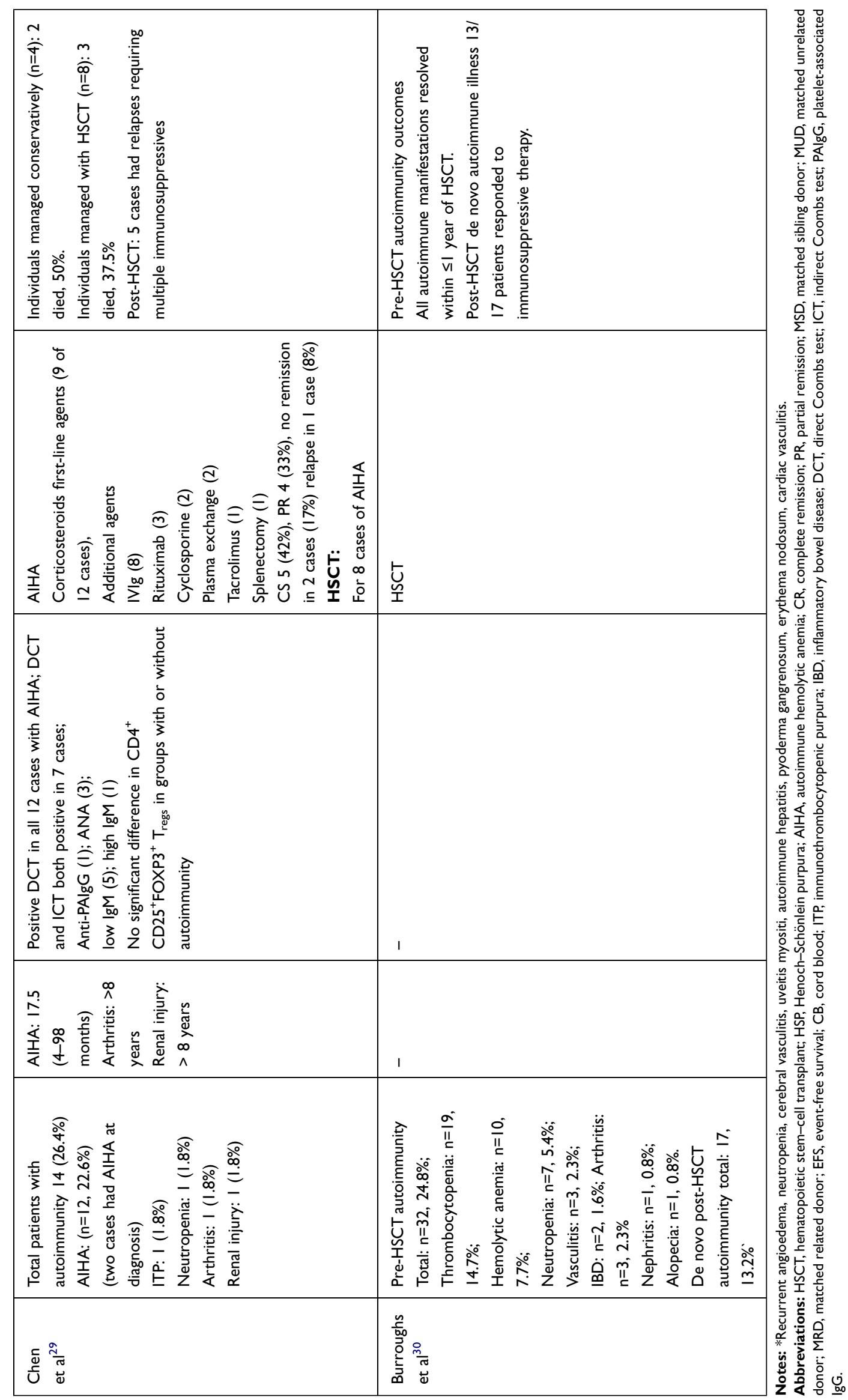


Pellier et al showed evidence of varicellazoster virus, Epstein-Barr virus, and human herpesvirus 6 in the aortic vessel wall on histopathology in a patient with WAS and aortic aneurysm. ${ }^{101}$

\section{Arthritis}

Arthritis has been reported in 1\%-29\% of patients with WAS, and accounts for $3 \%-52 \%$ of all autoimmune manifestations (Table 1). ${ }^{25,29,30,33,35,36}$ Sullivan et al observed arthritis in 32 of 152 patients (21\%). Of these, 17 had transient arthritis and 15 persistent arthritis. ${ }^{25}$ Median age at presentation of arthritis was 45.3 (13-180) months. ${ }^{33}$

\section{Renal Disease}

Onset of renal disease in XLT/WAS occurs at a relatively later age: $7-20$ years. ${ }^{36,37}$ Clinical manifestations include transient proteinuria, ${ }^{33}$ hematuria, azotemia, and nephritic-nephrotic syndrome. ${ }^{25,29,3335-37,100}$ Reported histopathological patterns include $\operatorname{IgA}$ nephropathy, membranoproliferative glomerulonephritis, mesangial proliferation, and interstitial nephritis. ${ }^{108-111}$ IgA nephropathy is the commonest renal disease, described in $27 \%-41 \%$ of patients in various long-term cohorts. ${ }^{25,35,36}$ Prevalence appears to be higher in patients with residual WASp expression. $^{35,36}$ Screening for renal involvement has been recommended in all patients with XLT/WAS.

Aberrant glycosylation of IgA is attributed as a cause for renal disease associated with WAS. Elevated levels of $\beta 1,6-N$-acetyl-glucosaminyl transferases have been documented in patients with WAS. This leads to aberrant $O$-glycosylation of sialophorin in patients with WAS. ${ }^{101,112}$

\section{Inflammatory Bowel Disease (IBD)}

Bleeding from the gastrointestinal tract is a common symptom in WAS and often secondary to thrombocytopenia. In our cohort, $49.4 \%$ of children presented with bloodstained stools. ${ }^{40}$ Autoimmune colitis has also been reported in 1\%-9\% of patients. ${ }^{25,29,30,35,36}$ Both ulcerative colitis and Crohn's disease have been observed. WASassociated colitis is challenging to treat and often refractory to immunosuppressants. ${ }^{113}$ In our experience, IBD can be a presenting feature of WAS. Ohya et al reported that $16.6 \%$ of patients with IBD had a $W A S$ mutation. ${ }^{113}$ Similarly, Cannioto et al showed that $25 \%$ of patients aged $<2$ years with IBD were later shown to have WAS. ${ }^{114}$

WASp-deficient mice have been observed to have chronic colitis with mucosal thickening and lymphocytic and neutrophilic infiltrates in the lamina propria. ${ }^{115}$ Suggested pathogenic mechanisms for IBD in WAS include WASp deficiency-mediated dysfunction of $T_{\text {reg }}$ cells and anti-inflammatory macrophages, increased selfreactive $\mathrm{B}$ cells, and altered gut microbiota. ${ }^{113,116}$

\section{Other Rheumatic Manifestations}

Monteferrante et al described lupus nephritis in a patient with WAS. ${ }^{117}$ Similarly, other connective-tissue disorders like dermatomyositis, ${ }^{27}$ uveitis, ${ }^{27}$ autoimmune hepatitis, ${ }^{27}$ primary sclerosing cholangitis, ${ }^{40}$ amyloidosis, ${ }^{39}$ and relapsing polychondritis ${ }^{118}$ have been reported in the context of WAS. $^{27}$ Crestani et al reported positive antineutrophil cytoplasmic antibodies, and positive antiphospholipid antibodies in patients with WAS. ${ }^{119}$

\section{Autoimmune Skin Diseases}

Eczematous dermatitis is a cardinal clinical manifestation of WAS. Eosinophilia and elevated levels of serum IgE are associated findings. DC dysfunction and skewed $\mathrm{T}_{\mathrm{H}} 2$ immunity may play a role in the development of eczema in this condition. ${ }^{120}$ Apart from atopy, other autoimmune skin manifestations have been reported in WAS. These include recurrent angioedema, pyoderma gangrenosum, and erythema nodosum. ${ }^{25}$ Alopecia has been reported in $1 \%-3 \%$ of patients with WAS. ${ }^{25,30,34,36}$

\section{Posttransplant Autoimmune Manifestations}

Development of autoimmunity is considered a predictor of severe disease, and often warrants the need for early HSCT in patients with XLT/WAS. HSCT is curative and is associated with 5 -year survival of $91 \%{ }^{30}$ However, occurrence of autoimmunity in the posttransplant period has been observed in $13 \%-20 \%$ of patients (Table 4$){ }^{26-30}$ Autoimmune cytopenia is the commonest autoimmune manifestation seen after HSCT. ${ }^{30}$ Burroughs et al reported that $75 \%$ of patients with autoimmune manifestations responded to immunosuppressive therapy and attained remission within a year of transplant. ${ }^{30}$ Mixed or split donor chimerism is an important predictor of development of post-HSCT autoimmunity. ${ }^{26,27,30}$ Autoimmunity is most frequently encountered in patients who undergo matched unrelated donor transplants. However, it is also seen in matched related-donor and matched sibling-donor transplants. $^{26,30}$ Presence of pretransplant autoimmune 
Table 4 Studies reporting posttransplant autoimmunity in XLT/WAS

\begin{tabular}{|c|c|c|}
\hline & Autoimmune manifestations, n (\%) & Treatment/outcome \\
\hline $\begin{array}{l}\text { Ozsahin } \\
\text { et } \mathrm{al}^{26}\end{array}$ & $\begin{array}{l}\text { Autoimmunity after HSCT Total 19/96, } 19.7 \% \\
\text { Thrombocytopenia: } n=10,10.4 \% \\
\text { AlHA: } n=3,3 \% \\
\text { Neutropenia: } n=1,1 \% \\
\text { Vasculitis: } n=2,2 \% \\
\text { IBD: } n=1,1 \% \\
\text { Pericarditis: } n=I, I \% \\
\text { Addison's disease: } n=1,1 \% \text { Autoimmune thyroiditis: } n=1,1 \%\end{array}$ & $\begin{array}{l}\text { Risk factors of autoimmunity after HSCT } \\
\text { MUD: } 9 / 32,28 \% \\
\text { MRD: } 5 / 19,26 \% \\
\text { MSD: } 5 / 45,11 \%(p=0.04) \\
\text { Pre-HSCT autoimmunity: } 7 / 17 \text { had persisted autoimmunity } \\
\text { after HSCT, though not significant }(p=0.06) \\
\text { Mixed/split donor chimerism: } p<0.00 \text { I }\end{array}$ \\
\hline $\begin{array}{l}\text { Moratto } \\
\text { et } \mathrm{al}^{27}\end{array}$ & $\begin{array}{l}\text { Autoimmunity after HSCT } \\
25(12.7 \%)\end{array}$ & $\begin{array}{l}\text { Risk factors for autoimmunity after HSCT } \\
\text { Mixed chimerism of T cells }(p<0.05) \\
\text { Mixed chimerism of B cells }(p<0.05) \\
\text { Mixed chimerism of myeloid cells }(p<0.0 \mathrm{I}) \\
\text { No change in posttransplant mortality rate compared to } \\
\text { individuals with no autoimmunity }\end{array}$ \\
\hline Shin et $\mathrm{al}^{28}$ & 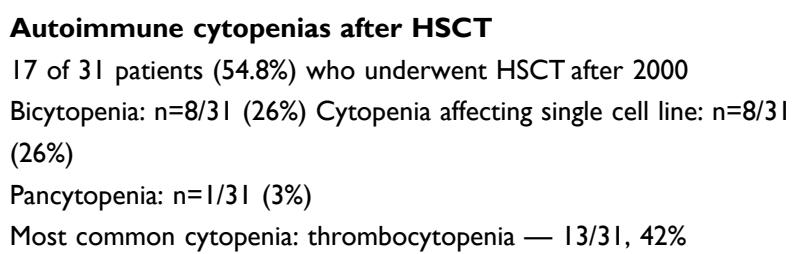 & $\begin{array}{l}\text { Median time to develop autoimmune cytopenia I48 (33- } \\
467) \text { days } \\
\text { Complete resolution (CR) of cytopenia: } 1 \text { I/I7, } 67 \% \text {. } \\
\text { Median time to achieve CR I.I ( } 0.35-2.7) \text { years } \\
\text { No role of mixed chimerism in autoimmunity: } p=0.5\end{array}$ \\
\hline $\begin{array}{l}\text { Chen } \\
\text { et } \mathrm{al}^{29}\end{array}$ & $\begin{array}{l}\text { Eight patients with autoimmunity underwent HSCT: five developed } \\
\text { autoimmune manifestations after HSCT, three died }\end{array}$ & $\begin{array}{l}\text { Post-HSCT } \\
5 \text { cases had relapses requiring multiple immunosuppressives }\end{array}$ \\
\hline $\begin{array}{l}\text { Burroughs } \\
\text { et } \mathrm{al}^{30}\end{array}$ & $\begin{array}{l}\text { Autoimmunity after HSCT } \\
17(13.1 \%) \\
\text { Cytopenias were predominant Hemolytic anemia: } n=13,10 \% \\
\text { Thrombocytopenia: } n=6,4.6 \% \text { Neutropenia: } n=3,2.3 \%\end{array}$ & $\begin{array}{l}\text { Post-HSCT de novo autoimmune illness } \\
\text { I3/I7 patients responded to immunosuppressive therapy } \\
\text { Risk factors of post-HSCT autoimmunity } \\
\text { Pre-HSCT autoimmunity: no risk }(p=0.779) \\
\text { MSD: no autoimmunity } \\
\text { MUD: } 23 \% \text { risk } \\
\text { CB: } 9 \% \text { risk } \\
\text { Mixed-edonor chimerism at } 6 \text { months: } p=0.049\end{array}$ \\
\hline
\end{tabular}

Abbreviations: HSCT, hematopoietic stem cell transplant; AlHA, autoimmune hemolytic anemia; MSD, matched sibling donor; MUD, matched unrelated donor; MRD, matched related donor; $\mathrm{CR}$, complete remission; $\mathrm{CB}$, cord blood.

manifestations does not increase the risk of development of post-HSCT autoimmunity. ${ }^{30}$

\section{Is Autoimmunity in WAS a Poor Prognostic Marker? Age at Onset of Autoimmunity}

Mahalaoui et al evaluated a subgroup of 26 patients that had onset of autoimmunity before the age of 2 years in their cohort of 160 patients with WAS. ${ }^{96}$ The authors concluded that development of early autoimmunity predicted a severe refractory disease course and required early HSCT for survival.

\section{Autoimmunity and Risk of Malignancy}

Presence of autoimmunity also increases the risk for development of malignancy in patients with WAS. Sullivan et al observed that $25 \%$ of patients with a history of autoimmune disease developed malignancy compared to $5 \%$ of patients without autoimmunity. ${ }^{25}$ Sallah et al demonstrated that $18 \%$ of patients with AIHA developed lymphoreticular malignancy. ${ }^{121}$

\section{Treatment of Autoimmunity}

Currently, HSCT is the best curative therapy available for WAS. Early studies on HSCT in WAS reported effective 
reconstitution of lymphoid cells, but impaired platelet engraftment. ${ }^{122-124}$ Long-term overall survival in recipients of unrelated bone-marrow grafts is $70 \%-78 \%$. $^{26,125}$ However, recent studies have reported event-free survival with HLA-identical sibling bone-marrow grafts to be $88 \%$, with overall survival of $90 \%-95 \% .^{27,30,126}$. Results of unrelated- and alternative-donor HSCT have also greatly improved to $>90 \%$. $^{30,37}$ HSCT with TCR $\alpha \beta / \mathrm{CD} 19$ depletion or posttransplant cyclophosphamide therapy for haploidentical donors has shown promising results and new opportunities for successful curative therapy in WAS/XLT. ${ }^{127}$

Gene therapy is an evolving and promising alternative approach when a transplant is not feasible due to unavailability of matched donors. Gene therapy was attempted with a gibbon ape leukemia virus $\gamma$-retroviral vector in 2006. ${ }^{77,127}$ Though successful engraftment was reported, it was limited by development of leukemia due to insertional leukemogenesis. Subsequently, therapy with a lentiviral vector was attempted in 31 patients with WAS. This resulted in successful engraftment, discontinuation of intravenous immunoglobulin (IVIg), improvement in platelet counts, and reduction in infections. Resolution of autoimmune manifestations was also demonstrated with gene therapy; however, the rate of de novo autoimmunity posttransplant was comparable with HSCT. ${ }^{127}$

Chemoprophylaxis with antimicrobials and monthly VIg are often used for prevention of infections. Management is usually individualized based on disease severity. Treatment of autoimmune manifestations is challenging, and may require require immunosuppressive therapies. These agents can further increase the risk of infections. Corticosteroids remain the first-line therapy for all autoimmune manifestations. Corticosteroids can induce variable rates of remission in patients with AIHA (Table 2). Additional agents are needed in patients who do not respond or attain partial remission. Cyclophosphamide, azathioprine, IVIg, rituximab, cyclosporine, plasma exchange, and tacrolimus have been used with variable results.

High- dose IVIg and oral or parenteral corticosteroids are used as first-line agents in patients with AIT who have significant bleeds. Rituximab is used for refractory cases. Splenectomy significantly increases and often normalizes platelet counts in refractory thrombocytopenia. ${ }^{77,128,129}$ However, splenectomy is associated with increased risk of sepsis and necessitates lifelong antimicrobial prophylaxis. $^{129}$ Moreover, thrombocytopenia may recur after splenectomy in patients with WAS. ${ }^{33}$ Splenectomy is reserved for very severe cases with no prospects from other curative interventions. Severe AIT after splenectomy is usually treated with IVIg, high-dose steroids, azathioprine, and cyclophosphamide. A majority of patients with skin vasculitis, arthritis, IBD, and renal disease associated with WAS respond to standard immunosuppressive regimens containing steroids and cyclosporine. ${ }^{33}$

\section{Conclusion}

Autoimmune manifestations are well-recognized complications in WAS. Varied clinical manifestations have been associated with the syndrome. Autoimmune cytopenia is the commonest. Development of autoimmunity is a poor prognostic marker and a predictor of development of malignancy. Pathogenic mechanisms for autoimmunity are not clearly defined. Corticosteroids with or without additional immunosuppressive agents are needed for treatment of autoimmune manifestations. HSCT is curative, but there is a risk of development of posttransplant autoimmunity.

\section{Disclosure}

The authors report no conflicts of interest in this work.

\section{References}

1. Wiskott A. Familiarer, angeborener morbus werlhofii? Monatsschr Kinderheilkd. 1937;68:212-216.

2. Aldrich RA, Steinberg AG, Campbell DC. Pedigree demonstrating a sex-linked recessive condition characterized by draining ears, eczematoid dermatitis and bloody diarrhea. Pediatrics. 1954;13:133-139.

3. Ochs HD, Thrasher AJ. The Wiskott-Aldrich syndrome. J Allergy Clin Immunol. 2006;117(4):725-738. doi:10.1016/j.jaci.2006.02.005

4. Ochs HD, Filipovich AH, Veys P, Cowan MJ, Kapoor N. WiskottAldrich syndrome: diagnosis, clinical and laboratory manifestations, and treatment. Biol Blood Marrow Transplant. 2009;15 (1):84-90. doi:10.1016/j.bbmt.2008.10.007

5. Notarangelo LD, Miao CH, Ochs HD. Wiskott-Aldrich syndrome. Curr Opin Hematol. 2008;15(1):30-36. doi:10.1097/MOH. 0b013e3282f30448

6. Perry GS 3rd, Spector BD, Schuman LM, et al. The Wiskott-Aldrich syndrome in the United States and Canada (1892-1979). J Pediatr. 1980;97(1):72-78. doi:10.1016/s0022-3476(80)80133-8

7. Fasth A. Primary immunodeficiency disorders in Sweden: cases among children, 1974-1979. J Clin Immunol. 1982;2(2):86-92. doi:10.1007/BF00916891

8. Ryser O, Morell A, Hitzig WH. Primary immunodeficiencies in Switzerland: first report of the national registry in adults and children. J Clin Immunol. 1988;8(6):479-485. doi:10.1007/ BF00916954

9. Derry JM, Ochs HD, Francke U. Isolation of a novel gene mutated in Wiskott-Aldrich syndrome. Cell. 1994;78(4):635644. doi:10.1016/0092-8674(94)90528-2

10. Kwan SP, Lehner T, Hagemann T, et al. Localization of the gene for the Wiskott-Aldrich syndrome between two flanking markers, TIMP and DXS255, on Xp11.22-Xp11.3. Genomics. 1991;10 (1):29-33. doi:10.1016/0888-7543(91)90480-3 
11. Rivero-Lezcano OM, Marcilla A, Sameshima JH, Robbins KC. Wiskott-Aldrich syndrome protein physically associates with Nck through Src homology 3 domains. Mol Cell Biol. 1995;15 (10):5725-5731. doi:10.1128/mcb.15.10.5725

12. Symons M, Derry JM, Karlak B, et al. Wiskott-Aldrich syndrome protein, a novel effector for the GTPase CDC42Hs, is implicated in actin polymerization. Cell. 1996;84(5):723-734. doi:10.1016/ s0092-8674(00)81050-8

13. Miki H, Nonoyama S, Zhu Q, Aruffo A, Ochs HD, Takenawa T. Tyrosine kinase signaling regulates Wiskott-Aldrich syndrome protein function, which is essential for megakaryocyte differentiation. Cell Growth Differ. 1997;8:195-202.

14. Miki H, Takenawa T. Direct binding of the verprolin-homology domain in N-WASP to actin is essential for cytoskeletal reorganization. Biochem Biophys Res Commun. 1998;243(1):73-78. doi:10.1006/bbrc. 1997.8064

15. Parolini O, Berardelli S, Riedl E, et al. Expression of Wiskott-Aldrich syndrome protein (WASP) gene during hematopoietic differentiation. Blood. 1997;1(90):70-75. doi:10.1182/blood.V90.1.70

16. Cory GO, MacCarthy-Morrogh L, Banin S, et al. Evidence that the Wiskott-Aldrich syndrome protein may be involved in lymphoid cell signaling pathways. J Immunol. 1996;157:3791-3795.

17. Gallego MD, Santamaría M, Peña J, Molina IJ. Defective actin reorganization and polymerization of Wiskott-Aldrich T cells in response to CD3-mediated stimulation. Blood. 1997;90(8):30893097. doi:10.1182/blood.V90.8.3089

18. Krawczyk C, Bachmaier K, Sasaki T, et al. Cbl-b is a negative regulator of receptor clustering and raft aggregation in $\mathrm{T}$ cells. Immunity. 2000;13(4):463-473. doi:10.1016/s1074-7613(00)00046-7

19. Sasahara Y, Rachid R, Byrne MJ, et al. Mechanism of recruitment of WASP to the immunological synapse and of its activation following TCR ligation. Mol Cell. 2002;10(6):1269-1281. doi:10.1016/s1097-2765(02)00728-1

20. Dupré L, Aiuti A, Trifari S, et al. Wiskott-Aldrich syndrome protein regulates lipid raft dynamics during immunological synapse formation. Immunity. 2002;17(2):157-166. doi:10.1016/ s1074-7613(02)00360-6

21. Linder S, Higgs $H$, Hüfner $K$, Schwarz $K$, Pannicke U, Aepfelbacher M. The polarization defect of Wiskott-Aldrich syndrome macrophages is linked to dislocalization of the Arp2/3 complex. J Immunol. 2000;165(1):221-225. doi:10.4049/ jimmunol.165.1.221

22. Jones GE, Zicha D, Dunn GA, Blundell M, Thrasher A Restoration of podosomes and chemotaxis in Wiskott-Aldrich syndrome macrophages following induced expression of WASp. Int J Biochem Cell Biol. 2002;34(7):806-815. doi:10.1016/s13572725(01)00162-5

23. Devriendt K, Kim AS, Mathijs G, et al. Constitutively activating mutation in WASP causes X-linked severe congenital neutropenia. Nat Genet. 2001;27(3):313-317. doi:10.1038/85886

24. Ancliff PJ, Blundell MP, Cory GO, et al. Two novel activating mutations in the Wiskott-Aldrich syndrome protein result in congenital neutropenia. Blood. 2006;108(7):2182-2189. doi:10.1182/ blood-2006-01-010249

25. Sullivan KE, Mullen CA, Blaese RM, Winkelstein JA. A multiinstitutional survey of the Wiskott-Aldrich syndrome. J Pediatr. 1994;125(6):876-885. doi:10.1016/s0022-3476(05)82002-5

26. Ozsahin H, Le Deist F, Benkerrou M, et al. Bone marrow transplantation in 26 patients with Wiskott-Aldrich syndrome from a single center. J Pediatr. 1996;129(2):238-244. doi:10.1016/ s0022-3476(96)70248-2

27. Moratto D, Giliani S, Bonfim C, et al. Long-term outcome and lineage-specific chimerism in 194 patients with Wiskott-Aldrich syndrome treated by hematopoietic cell transplantation in the period 1980-2009: an International Collaborative Study. Blood. 2011;118(6):1675-1684. doi:10.1182/blood-2010-11-319376
28. Shin CR, Kim MO, Li D, et al. Outcomes following hematopoietic cell transplantation for Wiskott-Aldrich syndrome. Bone Marrow Transplant. 2012;47(11):1428-1435. doi:10.1038/bmt.2012.31

29. Chen N, Zhang ZY, Liu DW, Liu W, Tang XM, Zhao XD. The clinical features of autoimmunity in 53 patients with Wiskott-Aldrich syndrome in China: a Single-Center Study. Eur J Pediatr. 2015;174 (10):1311-1318. doi:10.1007/s00431-015-2527-3

30. Burroughs LM, Petrovic A, Brazauskas R, et al. Excellent outcomes following hematopoietic cell transplantation for WiskottAldrich syndrome: a PIDTC report. Blood. 2020;135(23):20942105. doi:10.1182/blood.2019002939

31. Catucci M, Castiello MC, Pala F, Bosticardo M, Villa A. Autoimmunity in wiskott-Aldrich syndrome: an unsolved enigma. Front Immunol. 2012;3:209. doi:10.3389/fimmu.2012.00209

32. Gershwin ME, Blaese RM, Steinberg AD, Wistar R Jr, Strober W. Antibodies to nucleic acids in congenital immune deficiency states. J Pediatr. 1976;89(3):377-381. doi:10.1016/s0022-3476 (76)80531-8

33. Dupuis-Girod S, Medioni J, Haddad E, et al. Autoimmunity in Wiskott-Aldrich syndrome: risk factors, clinical features, and outcome in a single-center cohort of 55 patients. Pediatrics. 2003;111(5):e622-7. doi:10.1542/peds.111.5.e622

34. Imai K, Morio T, Zhu Y, et al. Clinical course of patients with WASP gene mutations. Blood. 2004;103(2):456-464. Epub 2003 Sep 11. PMID: 12969986. doi:10.1182/blood-2003-05-1480

35. Lee PP, Chen TX, Jiang L-P, et al. Clinical and molecular characteristics of 35 Chinese children with Wiskott-Aldrich syndrome. J Clin Immunol. 2009;29(4):490-500. doi:10.1007/ s10875-009-9285-9

36. Albert MH, Bittner TC, Nonoyama S, et al. X-linked thrombocytopenia (XLT) due to WAS mutations: clinical characteristics, long-term outcome, and treatment options. Blood. 2010;115 (16):3231-3238. doi:10.1182/blood-2009-09-239087

37. Elfeky RA, Furtado-Silva JM, Chiesa R, et al. One hundred percent survival after transplantation of 34 patients with Wiskott-Aldrich syndrome over 20 years. J Allergy Clin Immunol. 2018;142 (5):1654-1656.e7. doi:10.1016/j.jaci.2018.06.042

38. Jin YY, Wu J, Chen TX, Chen J. When WAS gene diagnosis is needed: seeking clues through comparison between patients with wiskottaldrich syndrome and idiopathic thrombocytopenic purpura? Front Immunol. 2019;10:1549. doi:10.3389/fimmu.2019.01549

39. Haskoloğlu Ş, Öztürk A, Öztürk G, et al. Clinical features and outcomes of 23 patients with Wiskott-Aldrich syndrome: a singlecenter experience. Turk J Haematol. 2020;37(4):271-281. doi:10.4274/tjh.galenos.2020.2020.0334

40. Suri D, Rikhi R, Jindal AK, et al. Wiskott Aldrich syndrome: a multi-institutional experience from India. Front Immunol. 2021;12:627651. doi:10.3389/fimmu.2021.627651

41. Malinova D, Fritzsche M, Nowosad CR, et al. WASp-dependent actin cytoskeleton stability at the dendritic cell immunological synapse is required for extensive, functional $\mathrm{T}$ cell contacts. J Leukoc Biol. 2016;99(5):699-710. doi:10.1189/ jlb.2A0215-050RR

42. Bouma G, Mendoza-Naranjo A, Blundell MP, et al. Cytoskeletal remodeling mediated by WASp in dendritic cells is necessary for normal immune synapse formation and T-cell priming. Blood. 2011;118(9):2492-2501. doi:10.1182/blood-2011-03-340265

43. Stabile H, Carlino C, Mazza C, et al. Impaired NK-cell migration in WAS/XLT patients: role of Cdc42/WASp pathway in the control of chemokine-induced beta 2 integrin high-affinity state. Blood. 2010;115(14):2818-2826. doi:10.1182/blood-2009-07235804

44. Maillard MH, Cotta-de-almeida V, Takeshima F, et al. The Wiskott-Aldrich syndrome protein is required for the function of CD4+CD25+Foxp3+ regulatory T cells. J Exp Med. 2007;204 (2):381-391. doi:10.1084/jem.20061338 
45. Westerberg L, Larsson M, Hardy SJ, Fernández C, Thrasher AJ, Severinson E. Wiskott-Aldrich syndrome protein deficiency leads to reduced B-cell adhesion, migration, and homing, and a delayed humoral immune response. Blood. 2005;105(3):1144-1152. doi:10.1182/blood-2004-03-1003

46. Molina IJ, Kenney DM, Rosen FS, Remold-O’Donnell E. T cell lines characterize events in the pathogenesis of the Wiskott-Aldrich syndrome. J Exp Med. 1992;176(3):867-874. doi:10.1084/jem.176.3.867

47. Rivers E, Thrasher AJ. Wiskott-Aldrich syndrome protein: emerging mechanisms in immunity. Eur $J$ Immunol. 2017;47 (11):1857-1866. doi:10.1002/eji.201646715

48. Massaad MJ, Ramesh N, Geha RS. Wiskott-Aldrich syndrome: a comprehensive review. Ann N Y Acad Sci. 2013;1285(1):26-43. doi:10.1111/nyas. 12049

49. Marangoni F, Bosticardo M, Charrier S, et al. Evidence for longterm efficacy and safety of gene therapy for Wiskott-Aldrich syndrome in preclinical models. Mol Ther. 2009;17(6):10731082. doi:10.1038/mt.2009.31

50. Golding B, Muchmore AV, Blaese RM. Newborn and WiskottAldrich patient B cells can be activated by TNP-Brucella abortus: evidence that TNP-Brucella abortus behaves as a T-independent type 1 antigen in humans. J Immunol. 1984;133:2966-2971. PMID: 6436369.

51. Meyer-Bahlburg A, Becker-Herman S, Humblet-Baron S, et al. Wiskott-Aldrich syndrome protein deficiency in B cells results in impaired peripheral homeostasis. Blood. 2008;112(10):41584169. doi:10.1182/blood-2008-02-140814

52. Erdei A, Isaák A, Török K, et al. Expression and role of CR1 and $\mathrm{CR} 2$ on $\mathrm{B}$ and $\mathrm{T}$ lymphocytes under physiological and autoimmune conditions. Mol Immunol. 2009;46(14):2767-2773. doi:10.1016/j.molimm.2009.05.181

53. Recher M, Burns SO, de la Fuente MA, et al. B cell-intrinsic deficiency of the Wiskott-Aldrich syndrome protein (WASp) causes severe abnormalities of the peripheral B-cell compartment in mice. Blood. 2012;119(12):2819-2828. doi:10.1182/blood-2011-09-379412

54. Castiello MC, Bosticardo M, Pala F, et al. Wiskott-Aldrich Syndrome protein deficiency perturbs the homeostasis of B-cell compartment in humans. J Autoimmun. 2014;50:42-50. doi:10. 1016/j.jaut.2013.10.006

55. Kolhatkar NS, Scharping NE, Sullivan JM, et al. B-cell intrinsic TLR7 signals promote depletion of the marginal zone in a murine model of Wiskott-Aldrich syndrome. Eur J Immunol. 2015;45 (10):2773-2779. doi:10.1002/eji.201545644

56. Simon KL, Anderson SM, Garabedian EK, Moratto D, Sokolic RA, Candotti F. Molecular and phenotypic abnormalities of B lymphocytes in patients with Wiskott-Aldrich syndrome. $J$ Allergy Clin Immunol. 2014;133(3):896-9.e4. doi:10.1016/j. jaci.2013.08.050

57. Bouma G, Carter NA, Recher M, et al. Exacerbated experimental arthritis in Wiskott-Aldrich syndrome protein deficiency: modulatory role of regulatory B cells. Eur J Immunol. 2014;44 (9):2692-2702. doi:10.1002/eji.201344245

58. Mars LT, Araujo L, Kerschen P, et al. Invariant NKT cells inhibit development of the Th17 lineage. Proc Natl Acad Sci U S A. 2009;106 (15):6238-6243. Epub 2009. doi:10.1073/pnas.0809317106

59. Yang JQ, Wen X, Kim PJ, Singh RR. Invariant NKT cells inhibit autoreactive B cells in a contact- and CD1d-dependent manner. $J$ Immunol. 2011;186(3):1512-1520. doi:10.4049/jimmunol.1002373

60. Astrakhan A, Ochs HD, Rawlings DJ. Wiskott-Aldrich syndrome protein is required for homeostasis and function of invariant NKT cells. J Immunol. 2009;182(12):7370-7380. doi:10.4049/ jimmunol.0804256

61. Locci M, Draghici E, Marangoni F, et al. The Wiskott-Aldrich syndrome protein is required for iNKT cell maturation and function. J Exp Med. 2009;206(4):735-742. Epub 2009 Mar 23. doi:10.1084/jem.20081773
62. Rönnblom L. The type I interferon system in the etiopathogenesis of autoimmune diseases. Ups J Med Sci. 2011;116(4):227-237. doi:10.3109/03009734.2011.624649

63. Prete F, Catucci M, Labrada M, et al. Wiskott-Aldrich syndrome protein-mediated actin dynamics control type-I interferon production in plasmacytoid dendritic cells. J Exp Med. 2013;210 (2):355-374. doi:10.1084/jem.20120363

64. Nikolov NP, Shimizu M, Cleland S, et al. Systemic autoimmunity and defective Fas ligand secretion in the absence of the WiskottAldrich syndrome protein. Blood. 2010;116(5):740-747. doi:10.1182/blood-2009-08-237560

65. Watanabe-Fukunaga R, Brannan CI, Copeland NG, Jenkins NA, Nagata S. Lymphoproliferation disorder in mice explained by defects in Fas antigen that mediates apoptosis. Nature. 1992;356 (6367):314-317. doi:10.1038/356314a0

66. Sobel ES, Kakkanaiah VN, Cohen PL, Eisenberg RA. Correction of gld autoimmunity by co-infusion of normal bone marrow suggests that gld is a mutation of the Fas ligand gene. Int Immunol. 1993;5(10):1275-1278. doi:10.1093/intimm/5.10.1275

67. Lee PP, Lobato-Márquez D, Pramanik N, et al. Wiskott-Aldrich syndrome protein regulates autophagy and inflammasome activity in innate immune cells. Nat Commun. 2017;8(1):1576. PMID: 29146903; PMCID: PMC5691069. doi:10.1038/s41467-01701676-0

68. Jin Y, Mazza C, Christie JR, et al. Mutations of the WiskottAldrich syndrome protein (WASP): hotspots, effect on transcription, and translation and phenotype/genotype correlation. Blood. 2004;104(13):4010-4019. doi:10.1182/blood-2003-05-1592

69. Lee WI, Huang JL, Jaing TH, Wu KH, Chien YH, Chang KW. Clinical aspects and genetic analysis of taiwanese patients with wiskott-Aldrich syndrome protein mutation: the first identification of x-linked thrombocytopenia in the chinese with novel mutations. J Clin Immunol. 2010;30(4):593-601. doi:10.1007/s10875010-9381-x

70. Ferrua F, Cicalese MP, Galimberti S, et al. Lentiviral haemopoietic stem/progenitor cell gene therapy for treatment of WiskottAldrich syndrome: interim results of a non-randomised, openlabel, Phase 1/2 clinical study. Lancet Haematol. 2019;6(5): e239-e253. doi:10.1016/S2352-3026(19)30021-3

71. Braun CJ, Boztug K, Paruzynski A, et al. Gene therapy for WiskottAldrich syndrome-long-term efficacy and genotoxicity. Sci Transl Med. 2014;6(227):227ra33. doi:10.1126/scitranslmed.3007280

72. Kolluri R, Shehabeldin A, Peacocke M, et al. Identification of WASP mutations in patients with Wiskott-Aldrich syndrome and isolated thrombocytopenia reveals allelic heterogeneity at the WAS locus. Hum Mol Genet. 1995;4(7):1119-1126. doi:10. 1093/hmg/4.7.1119

73. Vignesh P, Suri D, Rawat A, et al. Sclerosing cholangitis and intracranial lymphoma in a child with classical Wiskott-Aldrich syndrome. Pediatr Blood Cancer. 2017;64(1):106-109. doi:10.10 $02 /$ pbc. 26196

74. Xie JW, Zhang ZY, Wu JF, et al. In vivo reversion of an inherited mutation in a Chinese patient with Wiskott-Aldrich syndrome. Hum Immunol. 2015;76(6):406-413. doi:10.1016/j.humimm.20 15.04.001

75. Wu J, Liu D, Tu W, Song W, Zhao X. T-cell receptor diversity is selectively skewed in T-cell populations of patients with WiskottAldrich syndrome. J Allergy Clin Immunol. 2015;135(1):209216. doi:10.1016/j.jaci.2014.06.025

76. Liu DW, Zhang ZY, Zhao Q, et al. Wiskott-Aldrich syndrome/Xlinked thrombocytopenia in China: clinical characteristic and genotype-phenotype correlation. Pediatr Blood Cancer. 2015;62 (9):1601-1608. doi:10.1002/pbc.25559

77. Boztug K, Schmidt M, Schwarzer A, et al. Stem-cell gene therapy for the Wiskott-Aldrich syndrome. N Engl J Med. 2010;363 (20):1918-1927. doi:10.1056/NEJMoa1003548 
78. Hacein-Bey Abina S, Gaspar HB, Blondeau J, et al. Outcomes following gene therapy in patients with severe Wiskott-Aldrich syndrome. JAMA. 2015;313(15):1550-1563. doi:10.1001/jama.2015.3253

79. Buchbinder D, Nugent DJ, Fillipovich AH. Wiskott-Aldrich syndrome: diagnosis, current management, and emerging treatments. Appl Clin Genet. 2014;7:55-66. doi:10.2147/TACG.S58444

80. Pala F, Morbach H, Castiello MC, et al. Lentiviral-mediated gene therapy restores B cell tolerance in Wiskott-Aldrich syndrome patients. J Clin Invest. 2015;125(10):3941-3951. doi:10.1172/ JCI82249

81. Amarinthnukrowh $\mathrm{P}$, Ittiporn S, Tongkobpetch S, et al. Clinical and molecular characterization of Thai patients with WiskottAldrich syndrome. Scand $J$ Immunol. 2013;77(1):69-74. doi:10.1111/sji.12004

82. Schindelhauer D, Weiss M, Hellebrand H, et al. Wiskott-Aldrich syndrome: no strict genotype-phenotype correlations but clustering of missense mutations in the amino-terminal part of the WASP gene product. Hum Genet. 1996;98(1):68-76. doi:10.10 07/s004390050162

83. Knight T, Kotz K, Savaşan S. Autoimmune thyroiditis following HLA-matched sibling hematopoietic stem cell transplantation for Wiskott-Aldrich syndrome. Pediatr Transplant. 2018;22(5): e13222. doi:10.1111/petr.13222

84. Kolhatkar NS, Brahmandam A, Thouvenel CD, et al. Altered BCR and TLR signals promote enhanced positive selection of autoreactive transitional B cells in Wiskott-Aldrich syndrome. $J$ Exp Med. 2015;212(10):1663-1677. doi:10.1084/jem.20150585

85. Marangoni F, Trifari S, Scaramuzza S, et al. WASP regulates suppressor activity of human and murine CD4(+) CD25(+) FOXP3(+) natural regulatory T cells. J Exp Med. 2007;204 (2):369-380. doi:10.1084/jem.20061334

86. Glanzmann B, Möller M, Schoeman M, et al. Identification of a novel WAS mutation in a South African patient presenting with atypical Wiskott-Aldrich syndrome: a case report. BMC Med Genet. 2020;21(1):124. doi:10.1186/s12881-020-01054-6

87. Shigemura T, Nakazawa Y, Shimojo H, Kobayashi N, Agematsu $\mathrm{K}$. Immune complex-mediated glomerulonephritis in a patient with Wiskott-Aldrich syndrome. J Clin Immunol. 2016;36 (4):357-359. doi:10.1007/s10875-016-0258-5

88. Trifari S, Sitia G, Aiuti A, et al. Defective Th1 cytokine gene transcription in $\mathrm{CD} 4+$ and $\mathrm{CD} 8+\mathrm{T}$ cells from wiskott-aldrich syndrome patients. $J$ Immunol. 2006;177(10):7451-7461. doi:10.4049/jimmunol.177.10.7451

89. Du HQ, Zhang X, An YF, Ding Y, Zhao XD. Effects of WiskottAldrich syndrome protein deficiency on IL-10-producing regulatory B cells in humans and mice. Scand J Immunol. 2015;81 (6):483-493. doi:10.1111/sji.12282

90. Fillat C, Español T, Oset M, Ferrando M, Estivill X, Volpini V. Identification of WASP mutations in 14 Spanish families with Wiskott-Aldrich syndrome. Am J Med Genet. 2001;100(2):116121. doi:10.1002/ajmg.1228

91. Ochs HD, Slichter SJ, Harker LA, Von Behrens WE, Clark RA, Wedgwood RJ. The Wiskott-Aldrich syndrome: studies of lymphocytes, granulocytes, and platelets. Blood. 1980;55(2):243252. doi:10.1182/blood.V55.2.243.243

92. Gröttum KA, Hovig T, Holmsen H, Abrahamsen AF, Jeremic M, Seip M. Wiskott-Aldrich syndrome: qualitative platelet defects and short platelet survival. Br J Haematol. 1969;17(4):373-388. doi:10.1111/j.1365-2141.1969.tb01383.x

93. Baldini MG. Nature of the platelet defect in the Wiskott-Aldrich syndrome. Ann N Y Acad Sci. 1972;201(1):437-444. doi:10.1111/ j.1749-6632.1972.tb16316.x

94. Marathe BM, Prislovsky A, Astrakhan A, Rawlings DJ, Wan JY, Strom TS. Antiplatelet antibodies in WASP (-) mice correlate with evidence of increased in vivo platelet consumption. Exp Hematol. 2009;37(11):1353-1363. doi:10.1016/j.exphem.2009.08.007
95. Rivers E, Worth A, Thrasher AJ, Burns SO. How I manage patients with Wiskott Aldrich syndrome. $\mathrm{Br} J$ Haematol. 2019;185(4):647-655. doi:10.1111/bjh.15831

96. Mahlaoui N, Pellier I, Mignot C, et al. Characteristics and outcome of early-onset, severe forms of Wiskott-Aldrich syndrome. Blood. 2013;121(9):1510-1516. Epub 2012 Dec 20. doi:10.1182/ blood-2012-08-448118

97. Johnston SL, Unsworth DJ, Dwight JF, Kennedy CT. Wiskott-Aldrich syndrome, vasculitis and critical aortic dilatation. Acta Paediatr. 2001;90(11):1346-1348. doi:10.1080/080352501317130452

98. McCluggage WG, Armstrong DJ, Maxwell RJ, Ellis PK, McCluskey DR. Systemic vasculitis and aneurysm formation in the Wiskott-Aldrich syndrome. J Clin Pathol. 1999;52(5):390392. doi:10.1136/jcp.52.5.390

99. Nozicka Z, Parízková E, Bednárová J, Lichý J. Izolovaná arteritida bazálních mozkových tepen $\mathrm{u}$ dítěte $\mathrm{s}$ primární imunodeficiencí podmíněnou Wiskottovým-Aldrichovým syndromem [Isolated arteritis in the basal cerebral arteries in a child with primary immunodeficiency due to the Wiskott-Aldrich syndrome]. Cesk Patol. 1987;23:215-221. Czech. PMID: 3442841.

100. Lao YL, Wong SN, Lawton WM. Takayasu's arteritis associated with Wiskott-Aldrich syndrome. J Paediatr Child Health. 1992;28(5):407-409. doi:10.1111/j.1440-1754.1992. tb02703.x

101. Pellier I, Dupuis Girod S, Loisel D, et al. Occurrence of aortic aneurysms in 5 cases of Wiskott-Aldrich syndrome. Pediatrics. 2011;127(2):e498-504. doi:10.1542/peds.2009-2987

102. Watson RD, Gershwin ME, Smithwick E, Castles JJ, Ruebner B. Cutaneous $\mathrm{T}$ cell lymphoma and leukocytoclastic vasculitis in a long-term survivor of Wiskott-Aldrich syndrome. Ann Allergy. 1985;55:654-7703-5. PMID: 3877477.

103. Duzova A, Topaloglu R, Sanal O, et al. Henoch-Schönlein purpura in Wiskott-Aldrich syndrome. Pediatr Nephrol. 2001;16 (6):500-502. doi:10.1007/s004670100583

104. Kawakami C, Miyake M, Tamai H. Kawasaki disease in a patient with Wiskott-Aldrich syndrome: an increase in the platelet count. Int J Hematol. 2003;77(2):199-200. doi:10.10 07/BF02983223

105. Filipovich AH, Krivit W, Kersey JH, Burke BA. Fatal arteritis as a complication of Wiskott-Aldrich syndrome. J Pediatr. 1979;95 (5 Pt 1):742-744. PMID: 490243. doi:10.1016/s0022-3476(79) 80726-X

106. Longhurst HJ, Taussig D, Haque T, et al. Non-myeloablative bone marrow transplantation in an adult with Wiskott-Aldrich syndrome. $B r \quad J$ Haematol. 2002;116(2):497-499. doi:10.1046/ j.1365-2141.2002.03269.x

107. Cooper MD, Chae HP, Lowman JT, Krivit W, Good RA. WiskottAldrich syndrome. An immunologic deficiency disease involving the afferent limb of immunity. Am J Med. 1968;44(4):499-513. doi:10.1016/0002-9343(68)90051-x

108. Spitler LE, Wray BB, Mogerman S, Miller JJ 3rd, O’Reilly RJ, Lagios M. Nephropathy in the Wiskott-Aldrich syndrome. Pediatrics. 1980;66:391-398. PMID: 7422429.

109. Gutenberger J, Trygstad CW, Stiehm ER, Opitz JM, Thatcher LG, Bloodworth JM Jr. Familial thrombocytopenia, elevated serum IgA levels and renal disease. A report of a kindred. Am J Med. 1970;49(6):729-741. doi:10.1016/s0002-9343(70)80055-9

110. Webb MC, Andrews PA, Koffman CG, Cameron JS. Renal transplantation in Wiskott-Aldrich syndrome. Transplantation. 1993;56:1585. PMID: 8279047.

111. DeSanto NG, Sessa A, Capodicasa G, et al. IgA glomerulonephritis in Wiskott-Aldrich syndrome. Child Nephrol Urol. 19881989;9(1-2):118-120. PMID: 3251617.

112. Schurman SH, Candotti F. Autoimmunity in Wiskott-Aldrich syndrome. Curr Opin Rheumatol. 2003;15(4):446-453. doi:10.10 97/00002281-200307000-00012 
113. Ohya T, Yanagimachi M, Iwasawa K, et al. Childhood-onset inflammatory bowel diseases associated with mutation of Wiskott-Aldrich syndrome protein gene. World $J$ Gastroenterol. 2017;23(48):8544-8552. doi:10.3748/wjg.v23.i48.8544

114. Cannioto Z, Berti I, Martelossi S, et al. IBD and IBD mimicking enterocolitis in children younger than 2 years of age. Eur $J$ Pediatr. 2009;168(2):149-155. doi:10.1007/s00431-008-0721-2

115. Snapper SB, Rosen FS, Mizoguchi E, et al. Wiskott-Aldrich syndrome protein-deficient mice reveal a role for WASP in T but not B cell activation. Immunity. 1998;9(1):81-91. doi:10. 1016/s1074-7613(00)80590-7

116. Biswas A, Shouval DS, Griffith A, et al. WASP-mediated regulation of anti-inflammatory macrophages is IL-10 dependent and is critical for intestinal homeostasis. Nat Commun. 2018;9(1):1779. doi:10.1038/s41467-018-03670-6

117. Monteferrante G, Giani M, van den Heuvel M. Systemic lupus erythematosus and Wiskott-Aldrich syndrome in an Italian patient. Lupus. 2009;18(3):273-277. doi:10.1177/09612033080 95000

118. Adriani M, Aoki J, Horai R, et al. Impaired in vitro regulatory $\mathrm{T}$ cell function associated with Wiskott-Aldrich syndrome. Clin Immunol. 2007;124(1):41-48. doi:10.1016/j.clim.2007.02.001

119. Crestani E, Volpi S, Candotti F, et al. Broad spectrum of autoantibodies in patients with Wiskott-Aldrich syndrome and X-linked thrombocytopenia. J Allergy Clin Immunol. 2015;136 (5):1401-4.e1-3. doi:10.1016/j.jaci.2015.08.010

120. Taylor MD, Sadhukhan S, Kottangada P, et al. Nuclear role of WASp in the pathogenesis of dysregulated TH1 immunity in human Wiskott-Aldrich syndrome. Sci Transl Med. 2010;2 (37):37ra44. doi:10.1126/scitranslmed.3000813

121. Sallah S, Wan JY, Hanrahan LR. Future development of lymphoproliferative disorders in patients with autoimmune hemolytic anemia. Clin Cancer Res. 2001;7:791-794. PMID: 11309323.

122. Bach FH, Albertini RJ, Joo P, Anderson JL, Bortin MM. Bonemarrow transplantation in a patient with the Wiskott-Aldrich syndrome. Lancet. 1968;292(7583):1364-1366. doi:10.1016/ s0140-6736(68)92672-x
123. Kapoor N, Kirkpatrick D, Blaese RM, et al. Reconstitution of normal megakaryocytopoiesis and immunologic functions in Wiskott-Aldrich syndrome by marrow transplantation following myeloablation and immunosuppression with busulfan and cyclophosphamide. Blood. 1981;57(4):692-696. PMID: 7008865. doi:10.1182/blood.V57.4.692.692

124. Ochs HD, Lum LG, Johnson FL, Schiffman G, Wedgwood RJ, Storb R. Bone marrow transplantation in the Wiskott-Aldrich syndrome. Complete hematological and immunological reconstitution. Transplantation. 1982;34(5):284-288. doi:10.1097/000 07890-198211000-00009

125. Filipovich AH, Stone JV, Tomany SC, et al. Impact of donor type on outcome of bone marrow transplantation for Wiskott-Aldrich syndrome: Collaborative Study of the international bone marrow transplant registry and the national marrow donor program. Blood. 2001;97(6):1598-1603. doi:10.1182/blood.v97.6.1598

126. Ozsahin H, Cavazzana-Calvo M, Notarangelo LD, et al. Longterm outcome following hematopoietic stem-cell transplantation in Wiskott-Aldrich syndrome: collaborative study of the European society for immunodeficiencies and European group for blood and marrow transplantation. Blood. 2008;111(1):439-445. doi:10. 1182/blood-2007-03-076679

127. Mallhi KK, Petrovic A, Ochs HD. Hematopoietic stem cell therapy for Wiskott-Aldrich syndrome: improved outcome and quality of life. J Blood Med. 2021;12:435-447. doi:10.2147/JBM. S232650

128. Corash L, Shafer B, Blaese RM. Platelet-associated immunoglobulin, platelet size, and the effect of splenectomy in the WiskottAldrich syndrome. Blood. 1985;65(6):1439-1443. PMID: 3995178. doi:10.1182/blood.V65.6.1439.bloodjournal6561439

129. Mullen CA, Anderson KD, Blaese RM. Splenectomy and/or bone marrow transplantation in the management of the Wiskott-Aldrich syndrome: long-term follow-up of 62 cases. Blood. 1993;82 (10):2961-2966. PMID: 8219187. doi:10.1182/blood.V82.10. 2961.2961

\section{Publish your work in this journal}

The Application of Clinical Genetics is an international, peerreviewed open access journal that welcomes laboratory and clinical findings in the field of human genetics. Specific topics include: Population genetics; Functional genetics; Natural history of genetic disease; Management of genetic disease; Mechanisms of genetic disease;
Counselling and ethical issues; Animal models; Pharmacogenetics; Prenatal diagnosis; Dysmorphology. The manuscript management system is completely online and includes a very quick and fair peerreview system, which is all easy to use. Visit http://www.dovepress. com/testimonials.php to read real quotes from published authors. 